\title{
Superconformal $\mathcal{N}=3$ SYM Low-Energy Effective Action
}

\author{
I.L. Buchbinder, ${ }^{a}$ E.A. Ivanov, ${ }^{b}$ I.B. Samsonov ${ }^{c, 1}$ and B.M. Zupnik ${ }^{b}$ \\ ${ }^{a}$ Departamento de Fisica, UFJF, Juiz de Fora, MG, Brazil and \\ Department of Theoretical Physics, Tomsk State Pedagogical University, Tomsk 634061, Russia ${ }^{2}$ \\ ${ }^{b}$ Bogolubov Laboratory of Theoretical Physics, Joint Institute for Nuclear Research, Dubna, 141980 \\ Moscow Region, Russia \\ ${ }^{c}$ INFN, Sezione di Padova, via F. Marzolo 8, 35131 Padova, Italy \\ E-mail: joseph@tspu.edu.ru, eivanov@theor.jinr.ru, \\ samsonov@mph.phtd.tpu.ru, zupnik@theor.jinr.ru
}

ABSTRACT: We construct a manifestly $\mathcal{N}=3$ supersymmetric low-energy effective action of $\mathcal{N}=3$ super Yang-Mills theory. The effective action is written in the $\mathcal{N}=3$ harmonic superspace and respects the full $\mathcal{N}=3$ superconformal symmetry. On mass shell this action is responsible for the four-derivative terms in the $\mathcal{N}=4$ SYM effective action, such as $F^{4} / X^{4}$ and its supersymmetric completions, while off shell it involves also higherderivative terms. For constant Maxwell and scalar fields its bosonic part coincides, up to the $F^{6} / X^{8}$ order, with the bosonic part of the D3 brane action in the $A d S_{5} \times S^{5}$ background. We also argue that in the sector of scalar fields it involves the correctly normalized Wess-Zumino term with the implicit SU(3) symmetry.

KeYwORDS: Extended supersymmetry, Superspaces, Supersymmetric effective theories, Supersymmetric gauge theory

ARXIV EPRINT: 1111.4145

\footnotetext{
${ }^{1}$ On leave from Tomsk Polytechnic University, 634050 Tomsk, Russia.

${ }^{2}$ Permanent address.
} 


\section{Contents}

1 Introduction 1

$2 \mathcal{N}=3$ SYM setup $\quad 3$

2.1 Superfield strengths in $\mathcal{N}=3$ harmonic superspace 3

2.2 Superconformal transformations in $\mathcal{N}=3$ HSS 6

3 Superconformal effective action $\quad 8$

3.1 Non-superconformal $F^{4}$ term $\quad 8$

3.2 Scale and $\gamma_{5}$ invariant $F^{4} / X^{4}$ term 8

$\begin{array}{ll}3.3 \text { Complete } \mathcal{N}=3 \text { superconformal symmetry } & 11\end{array}$

$\begin{array}{ll}3.4 & \text { Independence of the choice of vacua } \\ \end{array}$

4 Component structure $\quad \mathbf{1 5}$

$\begin{array}{lll}4.1 F^{4} / X^{4} \text { term } & 15\end{array}$

$\begin{array}{lll}4.2 F^{6} / X^{8} \text { term } & 16\end{array}$

$\begin{array}{lll}4.3 & \text { A comment on the Wess-Zumino term } & 18\end{array}$

$\begin{array}{llr}5 & \text { Summary and discussion } & 19\end{array}$

$\begin{array}{ll}\text { A Derivation of scale and } \gamma_{5} \text { invariant effective action } & \mathbf{2 0}\end{array}$

$\begin{array}{ll}\text { B Harmonic integrals } & 22\end{array}$

C Wess-Zumino term $\quad 23$

C.1 Derivation from five-dimensions 23

$\begin{array}{lll}\text { C.2 Expansion around vacuum } & 25\end{array}$

D Effective equations of motion $\quad 26$

\section{Introduction}

It is well known that the superfield formulations of supersymmetric field theories, with the maximal number of the underlying supersymmetries being manifest and off-shell, are extremely useful for studying quantum aspects of these theories. In many cases, such formulations not only drastically reduce the amount of perturbative calculations, but also allow one to make certain conjectures about a possible structure of the final results prior to any calculation. The $\mathcal{N}=1$ superspace [1] is natural for $\mathcal{N}=1, d=4$ supersymmetric models, while the adequate superfield approach to $\mathcal{N}=2, d=4$ theories is offered by 
$\mathcal{N}=2$ harmonic superspace [2]. As for the renowned $\mathcal{N}=4$ SYM theory, no appropriate formulation of it in terms of unconstrained off-shell $\mathcal{N}=4$ superfields is known to date.

On shell, the $\mathcal{N}=4 \mathrm{SYM}$ theory is equivalent to the $\mathcal{N}=3$ SYM theory (see [2] and refs. therein). The latter possesses an unconstrained superfield formulation in $\mathcal{N}=3$ harmonic superspace [3, 4], such that three out of four supersymmetries of the original theory are manifest and off-shell within this framework. This approach proved to be very fruitful for establishing quantum finiteness of $\mathcal{N}=3 \mathrm{SYM}$ theory [5], as well as for constructing the $\mathcal{N}=3$ supersymmetric Born-Infeld theory [6]. ${ }^{1}$ The basic goal of the present paper is to provide an evidence that the $\mathcal{N}=3$ harmonic superspace approach is also useful for studying the low-energy effective actions in the $\mathcal{N}=3$ and $\mathcal{N}=4$ SYM theories.

It is known that the leading terms in the $\mathcal{N}=4 \mathrm{SYM}$ effective action in the $\mathcal{N}=2$ superfield formulation (which manifests only two out of four supersymmetries) are described by the non-holomorphic potential [8-16]. The hypermultiplet completion of the non-holomorphic potential, such that it ensures the on-shell $\mathcal{N}=4$ supersymmetry, was found in [17] (and further elaborated on in refs. [18-20]). The full action contains a scaleinvariant and $\mathrm{SU}(4)$ symmetric $F^{4} / X^{4}$ term, as well as some other terms related to this leading one by $\mathcal{N}=4$ supersymmetry. Here $F_{m n}$ is the Maxwell field strength and $X^{2}$ is the square of $\mathrm{SU}(4)$ invariant norm of scalar fields.

In the present paper we develop the $\mathcal{N}=3$ harmonic superspace description of the leading terms in the $\mathcal{N}=4 \mathrm{SYM}$ effective action to the order $F^{4} / X^{4}$. We seek this action as an integral over the analytic subspace of the $\mathcal{N}=3$ harmonic superspace, with the Lagrangian density being a local functional of the analytic superfield strengths without derivatives on them. We show that the requirements of the scale and $\gamma_{5}$ invariance uniquely fix the form of this functional. We check that the action constructed respects the full $\mathrm{SU}(2,2 \mid 3)$ superconformal symmetry and, in components, yields the $F^{4} / X^{4}$ term, where $X^{2}=\varphi^{i} \bar{\varphi}_{i}$ is the bilinear $\mathrm{SU}(3)$ (and in fact $\mathrm{SU}(4)$ ) invariant of the involved scalar fields.

We stress that the obtained action is essentially defined on the Coulomb branch of the theory, when the scalar fields acquire non-vanishing vevs, $c^{i}=\left\langle\varphi^{i}\right\rangle \neq 0$. These constants $c^{i}$ explicitly appear in the effective action, so that the effective Lagrangian is singular at $c^{i}=0$. However, we show that the action is in fact independent of any particular choice of $c^{i}, c^{i} \neq 0$. This is entirely analogous to what happens in the $\mathcal{N}=2$ harmonic superspace formulation of the $\mathcal{N}=2$ improved tensor multiplet model given in [21]. It was emphasized there that the presence of such constants in the action has a topological origin. In accord with this interpretation, the low-energy $\mathcal{N}=4 \mathrm{SYM}$ effective action contains a topological term given by the Wess-Zumino action for the scalar fields $[22,23]$. Therefore the presence of such constants in the effective Lagrangian is not surprising.

One of the advantages of the $\mathcal{N}=3$ superspace formulation is the possibility to go off shell due to the existence of unconstrained gauge prepotentials. Varying with respect to these prepotentials, we obtain the effective equations of motion corresponding to the effective action. Like in the non-scale-invariant $\mathcal{N}=3$ Born-Infeld theory [6], elimination of

\footnotetext{
${ }^{1}$ A possible scale-invariant generalization of the $\mathcal{N}=3$ Born-Infeld theory was discussed in [7].
} 
some of the auxiliary fields from the effective equations of motion allows one to reproduce not only $F^{4} / X^{4}$ term, but also the $F^{6} / X^{8}$ term in the effective action, which precisely matches with that appearing in the component expansion of the conformally-invariant Born-Infeld action. Therefore, the effective action obtained reproduces the worldvolume action of D3 brane on the $A d S_{5} \times S^{5}$ background up to the order $F^{6} / X^{8}$.

The paper is organized as follows. Section 2 contains a brief summary of the basic ingredients of the $\mathcal{N}=3$ harmonic superspace formalism. In particular, we give the representation of the $\mathcal{N}=3$ superconformal group $\mathrm{SU}(2,2 \mid 3)$ on the $\mathcal{N}=3 \mathrm{SYM}$ superfield strengths. In Section 3, employing the scale and $\gamma_{5}$ invariance, we derive the $\mathcal{N}=3 \mathrm{SYM}$ low-energy effective action and then show its invariance under the full $\mathcal{N}=3$ superconformal group. In Section 4 we derive the $F^{4} / X^{4}$ and $F^{6} / X^{8}$ component terms from the superfield action. The last Section is devoted to discussing some open problems deserving further study. In Appendices A and B we collect some technical details concerning the derivation of the superfield action and calculation of $\mathrm{SU}(3)$ harmonic integrals. A possible four-dimensional representation of the Wess-Zumino term with manifest SU(3) symmetry is discussed in the Appendix C. In Appendix D we demonstrate that the $\mathcal{N}=3$ superfield effective action proposed can be used for studying the effective superfield equations of motion.

Throughout the paper we follow the $\mathcal{N}=3$ superspace conventions employed in [7] and $[6]$.

\section{$2 \mathcal{N}=3$ SYM setup}

\subsection{Superfield strengths in $\mathcal{N}=3$ harmonic superspace}

The standard $\mathcal{N}=3$ superspace is parametrized by the coordinates $z^{M}=\left(x^{m}, \theta_{i}^{\alpha}, \bar{\theta}^{i \dot{\alpha}}\right)$, where $i=1,2,3$ is the $\mathrm{SU}(3)$ triplet index. Following [3, 4], we introduce the $\mathrm{SU}(3)$ harmonic variables $u_{i}^{I}=\left(u_{i}^{1}, u_{i}^{2}, u_{i}^{3}\right)$ and their conjugates, $\bar{u}_{I}^{i}=\left(\bar{u}_{1}^{i}, \bar{u}_{2}^{i}, \bar{u}_{3}^{i}\right)$, with the properties

$$
u_{i}^{I} \bar{u}_{J}^{i}=\delta_{J}^{I}, \quad u_{i}^{I} \bar{u}_{I}^{j}=\delta_{i}^{j}, \quad \varepsilon^{i j k} u_{i}^{1} u_{j}^{2} u_{k}^{3}=1 .
$$

These defining relations are the orthogonality and completeness conditions. The harmonic variables allow one to convert the small indices $i, j, \ldots$ on which the R-symmetry $\mathrm{SU}(3)$ group is linearly realized, into the capital indices, $I, J, \ldots$, which are inert under $\mathrm{SU}(3)$. For instance, we will make use of the projected Grassmann variables, $\theta_{I}^{\alpha}=\theta_{i}^{\alpha} \bar{u}_{I}^{i}, \bar{\theta}^{I \dot{\alpha}}=\bar{\theta}^{i \dot{\alpha}} u_{i}^{I}$. Some of these projected Grassmann variables parametrize the analytic subspace,

$$
\left\{\zeta_{A}, u\right\}=\left\{x_{A}^{m}, \theta_{2}^{\alpha}, \theta_{3}^{\alpha}, \bar{\theta}^{1 \dot{\alpha}}, \bar{\theta}^{2 \dot{\alpha}}, u\right\}, \quad x_{A}^{m}=x^{m}-i \theta_{1} \sigma^{m} \bar{\theta}^{1}+i \theta_{3} \sigma^{m} \bar{\theta}^{3} .
$$

The analytic superspace (2.2) is closed under the $\mathcal{N}=3$ supersymmetry [3, 4], and, hence, plays a role similar to that of usual chiral subspace in the $\mathcal{N}=1$ superspace [1] and of the $\mathcal{N}=2$ harmonic analytic superspace [2]. 
The harmonic projections of the covariant spinor derivatives ${ }^{2}$,

$$
D_{\alpha}^{i}=\frac{\partial}{\partial \theta_{i}^{\alpha}}+2 i \bar{\theta}^{i \dot{\alpha}} \frac{\partial}{\partial x^{\alpha \dot{\alpha}}}, \quad \bar{D}_{i \dot{\alpha}}=-\frac{\partial}{\partial \bar{\theta}^{i \dot{\alpha}}}-2 i \theta_{i}^{\alpha} \frac{\partial}{\partial x^{\alpha \dot{\alpha}}},
$$

are given by $D_{\alpha}^{I}=D_{\alpha}^{i} u_{i}^{I}$ and $\bar{D}_{I \dot{\alpha}}=\bar{D}_{i \dot{\alpha}} \bar{u}_{I}^{i}$. It is important that in the analytic coordinates (2.2) two of these six derivatives become short,

$$
D_{\alpha}^{1}=\frac{\partial}{\partial \theta_{1}^{\alpha}}, \quad \bar{D}_{3 \dot{\alpha}}=-\frac{\partial}{\partial \bar{\theta}^{3 \dot{\alpha}}},
$$

thus demonstrating that the $\mathcal{N}=3$ analytic superfields (i.e. those living on the analytic superspace (2.2)) can be covariantly defined by the Grassmann Cauchy-Riemann conditions,

$$
D_{\alpha}^{1} \Phi(z, u)=\bar{D}_{3 \dot{\alpha}} \Phi(z, u)=0 \quad \Rightarrow \quad \Phi(z, u)=\hat{\Phi}\left(\zeta_{A}, u\right) .
$$

The explicit expressions for the other four derivatives in the analytic basis can be found in the appendix of our previous paper [7], where the harmonic derivatives $D_{J}^{I}$ are also written down. Among these harmonic derivatives, $D_{2}^{1}, D_{3}^{2}$ and $D_{3}^{1}$ commute with (2.4) and so preserve the analyticity, while the remaining three $D_{1}^{2}, D_{2}^{3}, D_{1}^{3}$ do not. These six harmonic derivatives, together with the U(1) charges $S_{1}$ and $S_{2}$, form an su(3) algebra [24].

The conventional $\mathcal{N}=3 \mathrm{SYM}$ superfield strengths in the standard $\mathcal{N}=3$ superspace are described by the antisymmetric $\mathrm{SU}(3)$ tensor superfields $W^{i j}=-W^{j i}$. In the linearized approximation, these superfields obey the constraints [25],

$$
D_{\alpha}^{i} W_{j l}=\frac{1}{2}\left(\delta_{j}^{i} D_{\alpha}^{k} W_{k l}-\delta_{l}^{i} D_{\alpha}^{k} W_{k j}\right), \quad \bar{D}_{i \dot{\alpha}} W_{j k}+\bar{D}_{j \dot{\alpha}} W_{i k}=0,
$$

which eliminate all non-physical components in these superfields and put the physical ones on shell. Projecting these superfield strengths on the harmonic variables, we obtain the following six superfields,

$$
\begin{aligned}
& \bar{W}^{12}=u_{i}^{1} u_{j}^{2} \bar{W}^{i j}, \quad \bar{W}^{23}=u_{i}^{2} u_{j}^{3} \bar{W}^{i j}, \quad \bar{W}^{13}=u_{i}^{1} u_{j}^{3} \bar{W}^{i j}, \\
& W_{12}=\bar{u}_{1}^{i} \bar{u}_{2}^{j} W_{i j}, \quad W_{23}=\bar{u}_{2}^{i} \bar{u}_{3}^{j} W_{i j}, \quad W_{13}=\bar{u}_{1}^{i} \bar{u}_{3}^{j} W_{i j} .
\end{aligned}
$$

It is straightforward to find the harmonic projections of the constraints (2.6), which gives rise to a number of differential relations among the superfields (2.7). Consider, for instance, $\bar{W}^{12}$ and $W_{23}$. They obey the following (on-shell) constraints $[24]^{3}$ :

(i) First-order analyticity constraints,

$$
\begin{aligned}
& D_{\alpha}^{1} \bar{W}^{12}=D_{\alpha}^{2} \bar{W}^{12}=\bar{D}_{3 \dot{\alpha}} \bar{W}^{12}=0, \\
& D_{\alpha}^{1} W_{23}=\bar{D}_{2 \dot{\alpha}} W_{23}=\bar{D}_{3 \dot{\alpha}} W_{23}=0 ;
\end{aligned}
$$

\footnotetext{
${ }^{2}$ We use the following rules of converting the vector and bi-spinorial indices into each other, $x_{\alpha \dot{\alpha}}=$ $\left(\sigma^{m}\right)_{\alpha \dot{\alpha}} x_{m}, x_{m}=\frac{1}{2}\left(\tilde{\sigma}_{m}\right)^{\dot{\alpha} \alpha} x_{\alpha \dot{\alpha}}, \partial_{\alpha \dot{\alpha}}=\frac{1}{2}\left(\sigma^{m}\right)_{\alpha \dot{\alpha}} \partial_{m}, \partial_{m}=\left(\tilde{\sigma}_{m}\right)^{\dot{\alpha} \alpha} \partial_{\alpha \dot{\alpha}}$.

${ }^{3}$ The constraints $(2.8)-(2.10)$ can also be derived by quantizing a massless superparticle moving in the $\mathcal{N}=3$ harmonic superspace $[26]$.
} 
(ii) First-order harmonic shortness constraints,

$$
\begin{aligned}
& D_{1}^{2} \bar{W}^{12}=D_{2}^{1} \bar{W}^{12}=D_{3}^{2} \bar{W}^{12}=D_{3}^{1} \bar{W}^{12}=0 \\
& D_{2}^{1} W_{23}=D_{3}^{2} W_{23}=D_{3}^{1} W_{23}=D_{2}^{3} W_{23}=0
\end{aligned}
$$

(iii) Second-order Grassmann linearity constraints,

$$
\begin{aligned}
& \left(D^{3}\right)^{2} \bar{W}^{12}=\left(\bar{D}_{1}\right)^{2} \bar{W}^{12}=\left(\bar{D}_{2}\right)^{2} \bar{W}^{12}=\left(\bar{D}_{1} \bar{D}_{2}\right) \bar{W}^{12}=0, \\
& \left(D^{2}\right)^{2} W_{23}=\left(D^{3}\right)^{2} W_{23}=\left(D^{2} D^{3}\right) W_{23}=\left(\bar{D}_{1}\right)^{2} W_{23}=0 .
\end{aligned}
$$

Altogether, the constraints (2.8), (2.9) and (2.10) kill all non-physical (auxiliary) field components in $\bar{W}^{12}$ and $W_{23}$ and put the physical ones on shell ${ }^{4}$. As a result, the superfield strengths $\bar{W}^{12}$ and $W_{23}$ have the following component structure in the analytic coordinates $(2.2)$,

$$
\begin{aligned}
W_{23}= & \varphi^{1}+2 i \theta_{2}^{\alpha} \bar{\theta}^{2 \dot{\alpha}} \partial_{\alpha \dot{\alpha}} \varphi^{1}-4 i \theta_{2}^{\alpha} \bar{\theta}^{1 \dot{\alpha}} \partial_{\alpha \dot{\alpha}} \varphi^{2}-4 i \theta_{3}^{\alpha} \bar{\theta}^{1 \dot{\alpha}} \partial_{\alpha \dot{\alpha}} \varphi^{3} \\
& +4 i \theta_{2}^{\alpha} \theta_{3}^{\beta} F_{\alpha \beta}+\bar{\theta}^{1 \dot{\alpha}} \bar{\lambda}_{\dot{\alpha}}+\theta_{2}^{\alpha} \lambda_{3 \alpha}-\theta_{3}^{\alpha} \lambda_{2 \alpha} \\
& +2 i \theta_{2}^{\alpha} \bar{\theta}^{2 \dot{\alpha}} \bar{\theta}^{1 \dot{\beta}} \partial_{\alpha \dot{\alpha}} \bar{\lambda}_{\dot{\beta}}+2 i \theta_{2}^{\beta} \theta_{3}^{\alpha} \bar{\theta}^{2 \dot{\alpha}} \partial_{\alpha \dot{\alpha}} \lambda_{2 \beta}+4 i \theta_{2}^{\beta} \theta_{3}^{\alpha} \bar{\theta}^{1 \dot{\alpha}} \partial_{\alpha \dot{\alpha}} \lambda_{1 \beta} \\
& +8 \theta_{2}^{\alpha} \theta_{3}^{\beta} \bar{\theta}^{1 \dot{\alpha}} \bar{\theta}^{2 \dot{\beta}} \partial_{\alpha \dot{\alpha}} \partial_{\beta \dot{\beta}} \varphi^{3}, \\
\bar{W}^{12}= & \bar{\varphi}_{3}-2 i \theta_{2}^{\alpha} \bar{\theta}^{2 \dot{\alpha}} \partial_{\alpha \dot{\alpha}} \bar{\varphi}_{3}+4 i \theta_{3}^{\alpha} \bar{\theta}^{1 \dot{\alpha}} \partial_{\alpha \dot{\alpha}} \bar{\varphi}_{1}+4 i \theta_{3}^{\alpha} \bar{\theta}^{2 \dot{\alpha}} \partial_{\alpha \dot{\alpha}} \bar{\varphi}_{2} \\
& +4 i \bar{\theta}^{1 \dot{\alpha}} \bar{\theta}^{2 \dot{\beta}} \bar{F}_{\dot{\alpha} \dot{\beta}}+\theta_{3}^{\alpha} \lambda_{\alpha}-\bar{\theta}^{2 \dot{\alpha}} \bar{\lambda}_{\dot{\alpha}}^{1}+\bar{\theta}^{1 \dot{\alpha}} \bar{\lambda}_{\dot{\alpha}}^{2} \\
& +2 i \theta_{2}^{\alpha} \theta_{3}^{\beta} \bar{\theta}^{2 \dot{\alpha}} \partial_{\alpha \dot{\alpha}} \lambda_{\beta}+2 i \bar{\theta}^{1 \dot{\alpha}} \bar{\theta}^{2 \dot{\beta}} \theta_{2}^{\alpha} \partial_{\alpha \dot{\alpha}} \bar{\lambda}_{\dot{\beta}}^{2}+4 i \bar{\theta}^{1 \dot{\alpha}} \bar{\theta}^{2 \dot{\beta}} \theta_{3}^{\alpha} \partial_{\alpha \dot{\alpha}} \bar{\lambda}_{\dot{\beta}}^{3} \\
& +8 \bar{\theta}^{1 \dot{\alpha}} \bar{\theta}^{2 \dot{\beta}} \theta_{2}^{\alpha} \theta_{3}^{\beta} \partial_{\alpha \dot{\alpha}} \partial_{\beta \dot{\beta}} \bar{\varphi}_{1} .
\end{aligned}
$$

Here

$$
\varphi^{I}=u_{i}^{I} \varphi^{i}, \quad \bar{\varphi}_{I}=\bar{u}_{I}^{i} \bar{\varphi}_{i},
$$

and $\varphi^{i}$ is a triplet of physical scalars, $\square \varphi^{i}=0$. The four spinor fields are comprised by the $\mathrm{SU}(3)$ singlet $\lambda_{\alpha}$ and the triplet $\lambda_{I \alpha}=\bar{u}_{I}^{i} \lambda_{i \alpha}$ which obey free equations of motion, $\partial^{\alpha \dot{\alpha}} \lambda_{\alpha}=\partial^{\alpha \dot{\alpha}} \lambda_{i \alpha}=0$. The fields $F_{\alpha \beta}=F_{(\alpha \beta)}$ and $\bar{F}_{\dot{\alpha} \dot{\beta}}=\bar{F}_{(\dot{\alpha} \dot{\beta})}$ are spinorial components of the Maxwell field strength $F_{m n}=\partial_{m} A_{n}-\partial_{n} A_{m}, \partial^{m} F_{m n}=0$.

The crucial feature of the $\mathcal{N}=3$ harmonic superspace approach is that one can relax some of the constraints (2.8), (2.9), (2.10) and express the superfield strengths in terms of unconstrained off-shell gauge superfield potentials [6]. Consider the analytic superfields $V_{2}^{1}$ and $V_{3}^{2}, D_{\alpha}^{1}\left(V_{2}^{1}, V_{3}^{2}\right)=\bar{D}_{3 \dot{\alpha}}\left(V_{2}^{1}, V_{3}^{2}\right)=0$. They possess the following gauge transformations

$$
\delta V_{2}^{1}=i D_{2}^{1} \lambda, \quad \delta V_{3}^{2}=i D_{3}^{2} \lambda
$$

\footnotetext{
${ }^{4}$ Besides eqs. (2.9), the original constraints (2.6) imply some other relations of the first order in spinor derivatives, connecting $\bar{W}^{12}$ and $W_{23}$ with the remaining harmonic projections of $W_{k l}$ and $\bar{W}^{k l}$. These extra constraints can be used to deduce the second-order constraints (2.10) which, together with (2.8) and (2.9), form a closed set of the harmonic superspace constraints on $\bar{W}^{12}$ and $W_{23}$ [26].
} 
with $\lambda$ being an analytic gauge superfield parameter. Using these superfields, one constructs the non-analytic gauge potentials $V_{1}^{2}$ and $V_{2}^{3}$ as solutions of the zero-curvature equations [6],

$$
D_{2}^{1} V_{1}^{2}=D_{1}^{2} V_{2}^{1}, \quad D_{3}^{2} V_{2}^{3}=D_{2}^{3} V_{3}^{2} .
$$

Finally, the gauge-invariant superfield strengths $\bar{W}^{12}$ and $W_{23}$ can be expressed in terms of $V_{1}^{2}$ and $V_{2}^{3}$ as

$$
\bar{W}^{12}=-\frac{1}{4} D^{1 \alpha} D_{\alpha}^{1} V_{1}^{2}, \quad W_{23}=\frac{1}{4} \bar{D}_{3 \dot{\alpha}} \bar{D}_{3}^{\dot{\alpha}} V_{2}^{3} .
$$

It should be pointed out that the analyticity constraints (2.8) are valid off shell while the other constraints (2.9) and (2.10) put the superfield strengths on shell, except for the equations $D_{2}^{1} \bar{W}^{12}=0$ and $D_{3}^{2} W_{23}=0$ which are also satisfied off shell.

\subsection{Superconformal transformations in $\mathcal{N}=3 \mathrm{HSS}$}

The $\mathcal{N}=3$ superconformal group $\mathrm{SU}(2,2 \mid 3)$, besides the $\mathcal{N}=3$ super Poincaré transformations, contains dilatation (with the parameter $a$ ), $\gamma_{5}$-transformation (with the parameter $b$ ), conformal boosts (with the parameters $k_{\alpha \dot{\alpha}}$ ), conformal supersymmetry (with the parameters $\eta_{\alpha}^{i}, \bar{\eta}_{i \dot{\beta}}$ ) and $\mathrm{SU}(3)$ R-symmetry transformations (with the parameters $\lambda_{i}^{j}, \overline{\lambda_{i}^{j}}=-\lambda_{j}^{i}$, $\left.\lambda_{i}^{i}=0\right)$. The realization of this supergroup on the analytic coordinates (2.8) was found in $[27]$

$$
\begin{aligned}
\delta_{\mathrm{sc}} x_{A}^{\alpha \dot{\alpha}}= & a x_{A}^{\alpha \dot{\alpha}}+k_{\beta \dot{\beta}} x_{A}^{\alpha \dot{\beta}} x_{A}^{\beta \dot{\alpha}}-4 k_{\beta \dot{\beta}} \theta_{2}^{\beta} \bar{\theta}^{2 \dot{\alpha}} \theta_{2}^{\alpha} \bar{\theta}^{2 \dot{\beta}}+4 i x_{A}^{\alpha \dot{\beta}} \bar{\theta}^{1 \dot{\alpha}} \bar{u}_{1}^{i} \bar{\eta}_{i \dot{\beta}} \\
& +2 i x_{A-}^{\alpha \dot{\beta}} \bar{\theta}^{2 \dot{\alpha}} \bar{u}_{2}^{i} \bar{\eta}_{i \dot{\beta}}+4 i x_{A}^{\beta \dot{\alpha}} \theta_{3}^{\alpha} u_{i}^{3} \eta_{\beta}^{i}+2 i x_{A+}^{\beta \dot{\alpha}} \theta_{2}^{\alpha} u_{i}^{2} \eta_{\beta}^{i} \\
& -4 i \lambda_{i}^{j} \theta_{3}^{\alpha} \bar{\theta}^{1 \dot{\alpha}} u_{j}^{3} \bar{u}_{1}^{i}-2 i \lambda_{i}^{j} \theta_{2}^{\alpha} \bar{\theta}^{1 \dot{\alpha}} u_{j}^{2} \bar{u}_{1}^{i}-2 i \lambda_{i}^{j} \theta_{3}^{\alpha} \bar{\theta}^{2 \dot{\alpha}} u_{j}^{3} \bar{u}_{2}^{i}, \\
\delta_{\mathrm{sc}} \theta_{2}^{\alpha}= & (a / 2+i b) \theta_{2}^{\alpha}+k_{\beta \dot{\beta}} x_{A+}^{\alpha \dot{\beta}} \theta_{2}^{\beta}-4 i\left(\theta_{2}^{\alpha} u_{i}^{2}+\theta_{3}^{\alpha} u_{i}^{3}\right) \theta_{2}^{\beta} \eta_{\beta}^{i} \\
& +x_{A+}^{\alpha \dot{\beta}} \bar{u}_{2}^{i} \bar{\eta}_{\dot{\beta}}+\lambda_{i}^{j}\left(\theta_{2}^{\alpha} u_{j}^{2}+\theta_{3}^{\alpha} u_{j}^{3}\right) \bar{u}_{2}^{i} \\
\delta_{\mathrm{sc}} \theta_{3}^{\alpha}= & (a / 2+i b) \theta_{3}^{\alpha}+k_{\beta \dot{\beta}} x_{A-}^{\alpha \dot{\beta}} \theta_{3}^{\beta}-4 i \theta_{3}^{\alpha} \theta_{3}^{\beta} u_{i}^{3} \eta_{\beta}^{i}+x_{A-}^{\alpha \dot{\beta}} \bar{u}_{3}^{i} \bar{\eta}_{\dot{\beta} i}+\lambda_{i}^{j} \theta_{3}^{\alpha} u_{j}^{3} \bar{u}_{3}^{i}, \\
\delta_{\mathrm{sc}} \bar{\theta}^{1 \dot{\alpha}}= & (a / 2-i b) \bar{\theta}^{1 \dot{\alpha}}+k_{\beta \dot{\beta}} x_{A+}^{\beta \dot{\alpha}} \bar{\theta}^{1 \dot{\beta}}+4 i \bar{\theta}^{1 \dot{\beta}} \bar{\theta}^{1 \dot{\alpha}} \bar{u}_{1}^{i} \bar{\eta}_{\dot{\beta} i}+x_{A+}^{\beta \dot{\alpha}} u_{i}^{1} \eta_{\beta}^{i}-\lambda_{i}^{j} \bar{\theta}^{1 \dot{\alpha}} \bar{u}_{1}^{i} u_{j}^{1}, \\
\delta_{\mathrm{sc}} \bar{\theta}^{2 \dot{\alpha}}= & (a / 2-i b) \bar{\theta}^{2 \dot{\alpha}}+k_{\beta \dot{\beta}} x_{A-}^{\beta \dot{\alpha}} \bar{\theta}^{2 \dot{\beta}}+4 i \bar{\theta}^{2 \dot{\beta}}\left(\bar{\theta}^{1 \dot{\alpha}} \bar{u}_{1}^{i}+\bar{\theta}^{2 \dot{\alpha}} \bar{u}_{2}^{i}\right) \bar{\eta}_{\dot{\beta} i} \\
& +x_{A-}^{\beta \dot{\alpha}} u_{i}^{2} \eta_{\beta}^{i}-\lambda_{i}^{j}\left(\bar{\theta}^{1 \dot{\alpha}} \bar{u}_{1}^{i}+\bar{\theta}^{2 \dot{\alpha}} \bar{u}_{2}^{i}\right) u_{j}^{2},
\end{aligned}
$$

where $x_{A \pm}^{\alpha \dot{\alpha}}=x_{A}^{\alpha \dot{\alpha}} \pm 2 i \theta_{2}^{\alpha} \bar{\theta}^{2 \dot{\alpha}}$. For preserving the $\mathcal{N}=3$ harmonic analyticity, the harmonic variables should transform according to the rules,

$$
\begin{array}{ll}
\delta_{\mathrm{sc}} u_{i}^{1}=u_{i}^{2} \lambda_{2}^{1}+u_{i}^{3} \lambda_{3}^{1}, & \delta_{\mathrm{sc}} \bar{u}_{1}^{i}=0, \\
\delta_{\mathrm{sc}} u_{i}^{2}=u_{i}^{3} \lambda_{3}^{2}, & \delta_{\mathrm{sc}} \bar{u}_{2}^{i}=-\bar{u}_{1}^{i} \lambda_{2}^{1}, \\
\delta_{\mathrm{sc}} u_{i}^{3}=0, & \delta_{\mathrm{sc}} \bar{u}_{3}^{i}=-\bar{u}_{2}^{i} \lambda_{3}^{2}-\bar{u}_{1}^{i} \lambda_{3}^{1},
\end{array}
$$

where

$$
\lambda_{J}^{I}=-4 i k_{\beta \dot{\beta}} \theta_{J}^{\beta} \bar{\theta}^{I \dot{\beta}}-4 i\left(\bar{\eta}_{\dot{\beta} i} \bar{\theta}^{I \dot{\beta}} \bar{u}_{J}^{i}+\theta_{J}^{\beta} \eta_{\beta}^{i} u_{i}^{I}\right)+u_{i}^{I} \bar{u}_{J}^{j} \lambda_{j}^{i} .
$$

In this paper we will use the so-called passive form of superconformal transformations of superfields, when the variation is taken at different points, e.g., $\delta_{\mathrm{sc}} W \simeq W^{\prime}\left(x^{\prime}\right)-W(x)$. 
In such an approach, not only the superfields but also their derivatives, as well as the superspace measures, should be varied while computing the superconformal transformations of the superfield actions.

It is known [2] that the analytic measure $d \zeta\left(\begin{array}{l}33 \\ 11\end{array}\right) d u$ is invariant under (2.16) and (2.17),

$$
\operatorname{Ber} \frac{\partial\left(x_{A}^{\prime}, \theta^{\prime}, u^{\prime}\right)}{\partial\left(x_{A}, \theta, u\right)}=1 .
$$

Using the coordinate transformations (2.16) and (2.17), it is straightforward to find the superconformal variations of harmonic derivatives:

$$
\begin{array}{ll}
\delta_{\mathrm{sc}} D_{2}^{1}=-\lambda_{2}^{1} S_{1}, & \delta_{\mathrm{sc}} D_{1}^{2}=\left(\lambda_{1}^{1}-\lambda_{2}^{2}\right) D_{1}^{2}, \\
\delta_{\mathrm{sc}} D_{3}^{2}=-\lambda_{3}^{2} S_{2}, & \delta_{\mathrm{sc}} D_{2}^{3}=\left(\lambda_{2}^{2}-\lambda_{3}^{3}\right) D_{2}^{3}, \\
\delta_{\mathrm{sc}} D_{3}^{1}=\lambda_{2}^{1} D_{3}^{2}-\lambda_{3}^{2} D_{2}^{1}-\lambda_{3}^{1}\left(S_{1}+S_{2}\right), & \delta_{\mathrm{sc}} D_{1}^{3}=\left(\lambda_{1}^{1}-\lambda_{3}^{3}\right) D_{1}^{3}+\lambda_{1}^{2} D_{2}^{3}-\lambda_{2}^{3} D_{1}^{2}, \\
\delta_{\mathrm{sc}} D_{1}^{1}=\delta_{\mathrm{sc}} D_{2}^{2}=\delta_{\mathrm{sc}} D_{3}^{3}=0, & \delta_{\mathrm{sc}} S_{1}=\delta_{\mathrm{sc}} S_{2}=0 .
\end{array}
$$

Recall that the gauge covariant harmonic derivatives involve the gauge superfield prepotentials

$$
\nabla_{J}^{I}=D_{J}^{I}+i V_{J}^{I}
$$

Requiring the lengthened derivatives (2.21) to be superconformally covariant, with taking into account the transformations (2.20), implies the following transformation laws for the gauge prepotentials:

$$
\begin{array}{ll}
\delta_{\mathrm{sc}} V_{2}^{1}=0, & \delta_{\mathrm{sc}} V_{1}^{2}=\left(\lambda_{1}^{1}-\lambda_{2}^{2}\right) V_{1}^{2}, \\
\delta_{\mathrm{sc}} V_{3}^{2}=0, & \delta_{\mathrm{sc}} V_{2}^{3}=\left(\lambda_{2}^{2}-\lambda_{3}^{3}\right) V_{2}^{3}, \\
\delta_{\mathrm{sc}} V_{3}^{1}=\lambda_{2}^{1} V_{3}^{2}-\lambda_{3}^{2} V_{2}^{1}, & \delta_{\mathrm{sc}} V_{1}^{3}=\left(\lambda_{1}^{1}-\lambda_{3}^{3}\right) V_{1}^{3}+\lambda_{1}^{2} V_{2}^{3}-\lambda_{2}^{3} V_{1}^{2} .
\end{array}
$$

Note that the superconformal variations of the analytic gauge superfields $V_{2}^{1}, V_{3}^{2}$ and $V_{3}^{1}$ were earlier given in $[2,27]$, while the transformations of the non-analytic gauge superfields $V_{1}^{2}, V_{2}^{3}$ and $V_{1}^{3}$ were not presented before.

Using (2.16) and (2.17) it is also easy to find the superconformal transformations of the covariant spinor derivatives (2.4),

$$
\begin{aligned}
\delta_{\mathrm{sc}} D_{\alpha}^{1} & =\left(-a / 2-i b-\lambda_{1}^{1}\right) D_{\alpha}^{1}+B_{\alpha}^{\beta} D_{\beta}^{1}, \\
\delta_{\mathrm{sc}} \bar{D}_{3 \dot{\alpha}} & =\left(-a / 2+i b+\lambda_{3}^{3}\right) \bar{D}_{3 \dot{\alpha}}+\bar{B}_{\dot{\alpha}}^{\dot{\beta}} \bar{D}_{3 \dot{\beta}},
\end{aligned}
$$

where $\lambda_{1}^{1}$ and $\lambda_{3}^{3}$ were defined in (2.18) and

$$
\begin{aligned}
B_{\alpha}^{\beta} & =-k_{\alpha \dot{\beta}}\left(x_{A+}^{\beta \dot{\beta}}+4 i \theta_{1}^{\beta} \bar{\theta}^{1 \dot{\beta}}\right)-4 i \theta_{I}^{\beta} u_{j}^{I} \eta_{\alpha}^{j}, \\
\bar{B}_{\dot{\alpha}}^{\dot{\beta}} & =-k_{\beta \dot{\alpha}}\left(x_{A-}^{\beta \dot{\beta}}-4 i \theta_{3}^{\beta} \bar{\theta}^{3 \dot{\beta}}\right)-4 i \bar{\theta}^{I \dot{\beta}} \bar{u}_{I}^{j} \bar{\eta}_{\dot{\alpha} j} .
\end{aligned}
$$

It is worth pointing out that the spinor derivatives $D_{\alpha}^{1}$ and $\bar{D}_{3 \dot{\alpha}}$ are not mixed under the superconformal transformations.

Finally, using the variations of the gauge prepotentials (2.22) and derivatives (2.23), we can find the superconformal transformations of the superfield strengths (2.15),

$$
\delta_{\mathrm{sc}} W_{23}=A W_{23}, \quad \delta_{\mathrm{sc}} \bar{W}^{12}=\bar{A} \bar{W}^{12},
$$


where

$$
A=-a+2 i b+\lambda_{2}^{2}+\lambda_{3}^{3}+\bar{B}_{\dot{\alpha}}^{\dot{\alpha}}, \quad \bar{A}=-a-2 i b-\lambda_{1}^{1}-\lambda_{2}^{2}+B_{\alpha}^{\alpha} .
$$

One can check that the superfields $A$ and $\bar{A}$ are analytic,

$$
D_{\alpha}^{1}(A, \bar{A})=\bar{D}_{3 \dot{\alpha}}(A, \bar{A})=0 .
$$

Hence, the transformations (2.25) preserve analyticity.

\section{Superconformal effective action}

\subsection{Non-superconformal $F^{4}$ term}

The $\mathcal{N}=3$ supersymmetric completion of the fourth-order term in the Born-Infeld action was constructed in [6],

$$
S_{4}=\frac{1}{32} \int d \zeta\left(\begin{array}{l}
33 \\
11
\end{array}\right) d u \frac{\left(\bar{W}^{12} W_{23}\right)^{2}}{(\bar{\Lambda} \Lambda)^{2}} .
$$

Here $\Lambda$ is a coupling constant of dimension one in mass units, which is introduced to ensure the correct dimension of the integrand. The analytic measure is defined as follows $[6,7]$,

$$
d \zeta\left(\begin{array}{l}
33 \\
11
\end{array}\right)=\frac{1}{16^{2}} d^{4} x_{A}\left(D^{3}\right)^{2}\left(D^{2}\right)^{2}\left(\bar{D}_{1}\right)^{2}\left(\bar{D}_{2}\right)^{2} .
$$

The analytic measure is dimensionless, $\left[d \zeta\left(\begin{array}{l}33 \\ 11\end{array}\right) d u\right]=0$, and $\left[\bar{W}^{12}\right]=\left[W_{23}\right]=1$. With this normalization of the analytic measure, it is straightforward to check that, along with other component terms, the action (3.1) yields the standard $F^{4}$ term,

$$
S_{4}=\frac{1}{2} \int d^{4} x \frac{F^{2} \bar{F}^{2}}{(\bar{\Lambda} \Lambda)^{2}}+\ldots
$$

Consider now the superconformal variation of the action (3.1),

$$
\delta_{\mathrm{sc}} S_{4}=\frac{1}{16} \int d \zeta\left(\begin{array}{l}
33 \\
11
\end{array}\right) d u(A+\bar{A}) \frac{\left(\bar{W}^{12} W_{23}\right)^{2}}{(\bar{\Lambda} \Lambda)^{2}},
$$

where we have used the variations of the superfield strengths (2.25) and the invariance of the analytic measure (2.19). Here $A$ and $\bar{A}$ are superfields (2.26) collecting the constant parameters of the superconformal transformations (2.16) and (2.17). The variation (3.4) is non-zero, hence the action (3.1) is not superconformal.

\subsection{Scale and $\gamma_{5}$ invariant $F^{4} / X^{4}$ term}

Our aim here is to find a superconformal generalization of the action (3.1). In what follows we will denote this superconformal action by $\Gamma$ (to stress that it is a part of the $\mathcal{N}=3$ SYM low-energy effective action). The action $\Gamma$ should meet the following criteria:

1. It should be a local functional defined on the analytic superspace and constructed out of the superfield strengths $\bar{W}^{12}$ and $W_{23}$ without derivatives on them,

$$
\Gamma=\int d \zeta\left(\begin{array}{l}
33 \\
11
\end{array}\right) d u \mathcal{H}_{33}^{11}\left(\bar{W}^{12}, W_{23}\right) \text {. }
$$


The analytic Lagrangian density $\mathcal{H}_{33}^{11}$ is an arbitrary function of its arguments, such that its external harmonic $U(1)$ charges cancel those of the analytic integration measure. This is the most general form of the superspace action yielding terms with four-derivatives in components, since the analytic measure (3.2) contains just eight spinor derivatives which can produce four space-time ones on the component fields.

2. The action $\Gamma$ should be invariant under the superconformal transformations (2.25),

$$
\delta_{\mathrm{sc}} \Gamma=0 .
$$

As a weaker requirement, in this subsection we will employ only the scale- and $\gamma_{5^{-}}$ transformations out of the full $\mathrm{SU}(2,2 \mid 3)$ superconformal group. We will show that this is sufficient to uniquely specify the structure of the action. The check of the full superconformal symmetry will be performed in the next subsection.

3. In the component-field expansion the action $\Gamma$ should reproduce the scale- and $\mathrm{SU}(3)-$ invariant $F^{4} / X^{4}$ term (3.3),

$$
\int d^{4} x \frac{F^{2} \bar{F}^{2}}{\left(\varphi^{i} \bar{\varphi}_{i}\right)^{2}}
$$

4. We are interested in the low-energy effective action for massless fields, with massive ones being integrated out. The massive fields appear in the Coulomb branch when the gauge symmetry is broken down spontaneously. For instance, the $\mathrm{SU}(2)$ gauge symmetry is broken down to $\mathrm{U}(1)$ when the scalar field corresponding to the Cartan subalgebra of $\mathrm{su}(2)$ acquire non-trivial vevs,

$$
c^{i}=\left\langle\varphi^{i}\right\rangle \neq 0, \quad \bar{c}_{i}=\left\langle\bar{\varphi}_{i}\right\rangle \neq 0 .
$$

However, the effective action should be independent of any particular choice of these constants,

$$
\Gamma\left(c^{i}, \bar{c}_{j}^{\prime}\right)=\Gamma\left(c^{i}, \bar{c}_{j}\right), \quad c^{i} \bar{c}_{i} \neq 0,
$$

because such a dependence would break superconformal invariance of the action.

5. Finally, we simplify the problem by considering only that part of the action (3.5) which does not vanish on the mass shell, i.e., we will assume that the superfield strengths obey the constraints (2.8)-(2.10). We will neglect all terms in the action $\Gamma$ which vanish when these constraints are imposed. As a consequence, one is free to add to $\Gamma$ or to subtract from it the following expressions which vanish on the mass shell,

$$
\begin{aligned}
\int d \zeta\left(\begin{array}{l}
33 \\
11
\end{array}\right) \bar{W}^{12} \mathcal{F}\left(W_{23}\right) & \propto \int d^{4} x\left(D^{3}\right)^{2}\left(D^{2}\right)^{2}\left(\bar{D}_{1}\right)^{2}\left[\mathcal{F}\left(W_{23}\right)\left(\bar{D}_{2}\right)^{2} \bar{W}^{12}\right] \simeq 0 \\
\int d \zeta\left(\begin{array}{l}
33 \\
11
\end{array}\right) W_{23} \mathcal{F}\left(\bar{W}^{12}\right) & \propto \int d^{4} x\left(D^{3}\right)^{2}\left(\bar{D}_{2}\right)^{2}\left(\bar{D}_{1}\right)^{2}\left[\mathcal{F}\left(\bar{W}^{12}\right)\left(D^{2}\right)^{2} W_{23}\right] \simeq 0 .
\end{aligned}
$$

Here $\mathcal{F}(W)$ is an arbitrary function of its argument. We will frequently employ this property while deriving the action. 
Now we shall turn to constructing the action $\Gamma$ which obeys the requirements and properties listed above.

As the first step, we introduce the shifted scalar fields, $\phi^{i}$ and $\bar{\phi}_{i}$,

$$
\varphi^{i}=c^{i}+\phi^{i}, \quad \bar{\varphi}_{i}=\bar{c}_{i}+\bar{\phi}_{i}, \quad\left\langle\phi^{i}\right\rangle=\left\langle\bar{\phi}_{i}\right\rangle=0 .
$$

Next, we define the harmonic projections of these vev constants

$$
c^{1}=u_{i}^{1} c^{i}, \quad c^{2}=u_{i}^{2} c^{i} \quad c^{3}=u_{i}^{3} c^{i}, \quad \bar{c}_{1}=\bar{u}_{1}^{i} \bar{c}_{i}, \quad \bar{c}_{2}=\bar{u}_{2}^{i} \bar{c}_{i}, \quad \bar{c}_{3}=\bar{u}_{3}^{i} \bar{c}_{i} .
$$

Using these objects, we introduce the shifted superfield strengths, $\bar{\omega}^{12}$ and $\omega_{23}$,

$$
\bar{W}^{12}=\bar{c}_{3}+\bar{\omega}^{12}, \quad W_{23}=c^{1}+\omega_{23} .
$$

Under the scale and $\gamma_{5}$ transformations these shifted superfields transform inhomogeneously,

$$
\delta_{\mathrm{sc}} \bar{\omega}^{12}=\bar{A} \bar{c}_{3}+\bar{A} \bar{\omega}^{12}, \quad \delta_{\mathrm{sc}} \omega_{23}=A c^{1}+A \omega_{23},
$$

where $A=-a+2 i b$. The case of generic $A$ and $\bar{A}$ defined in (2.26) will be considered in the next subsection.

We point out that on shell, when the relations (3.10) are valid, the non-superconformal action (3.1) can be rewritten in terms of $\bar{\omega}^{12}$ and $\omega_{23}$ as

$$
S_{4}=\frac{1}{32} \int d \zeta\left(\begin{array}{l}
33 \\
11
\end{array}\right) d u \frac{\left(\bar{\omega}^{12} \omega_{23}\right)^{2}}{\left(c^{i} \bar{c}_{i}\right)^{2}} .
$$

Here we substituted $\left(c^{i} \bar{c}_{i}\right)^{2}$ in the denominator instead of $(\bar{\Lambda} \Lambda)^{2}$, because no other dimensionful constants besides the vevs $c^{i}$ can be present in the superconformal case.

We search for a superconformal generalization of the action (3.15) in the form

$$
\Gamma=\frac{\alpha}{8} \int d \zeta\left(\begin{array}{l}
33 \\
11
\end{array}\right) d u \frac{\left(\bar{\omega}^{12} \omega_{23}\right)^{2}}{\left(c^{i} \bar{c}_{i}\right)^{2}} H\left(\frac{\bar{\omega}^{12} c^{3}}{c^{i} \bar{c}_{i}}, \frac{\omega_{23} \bar{c}_{1}}{c^{i} \bar{c}_{i}}\right)
$$

where $H(x, y)$ is some function to be determined and $\alpha$ is a dimensionless coupling constat. The arguments $\frac{\bar{\omega}^{12} c^{3}}{c^{i} \bar{c}_{i}}$ and $\frac{\omega_{23} \bar{c}_{1}}{c^{i} \bar{c}_{i}}$ of the function $H$ are chargeless and dimensionless. We assume that the function $H$ has a regular power expansion with respect to its arguments,

$$
H(x, y)=\sum_{m, n=0}^{\infty} \alpha_{m, n} x^{m} y^{n}
$$

with undefined coefficients $\alpha_{m, n}$. The reality of the action (3.16) under complex conjugation implies the symmetry of this function, $H(x, y)=H(y, x)$, whence $\alpha_{m, n}=\alpha_{n, m}$.

Reordering the summation in (3.17), it is convenient to represent (3.16) as

$$
\Gamma=\sum_{n=0}^{\infty} \Gamma_{n}, \quad \Gamma_{n}=\frac{\alpha}{8} \int d \zeta\left(\begin{array}{l}
33 \\
11
\end{array}\right) d u \frac{\left(\bar{\omega}^{12} \omega_{23}\right)^{2}}{\left(c^{i} \bar{c}_{i}\right)^{2}} \sum_{i=0}^{n} \alpha_{i, n-i}\left(\frac{\bar{\omega}^{12} c^{3}}{c^{i} \bar{c}_{i}}\right)^{i}\left(\frac{\omega_{23} \bar{c}_{1}}{c^{i} \bar{c}_{i}}\right)^{n-i} .
$$

The invariance of the action (3.18) under the transformations (3.14) can be ensured order by order, i.e., the non-vanishing terms from $\delta_{\mathrm{sc}} \Gamma_{n}$ are required to be cancelled by similar 
terms from $\delta_{\mathrm{sc}} \Gamma_{n+1}$, and so forth. This recurrence procedure imposes severe restrictions on the coefficients $\alpha_{m, n}$. The technical details of this procedure are given in the Appendix A, with the following result:

$$
\alpha_{m, n}=(-1)^{m+n} \frac{(m+n+2) !}{(n+2) n !(m+2) m !} .
$$

With these coefficients, the series (3.17) can be summed up as follows,

$$
H(x, y)=\frac{\ln (1+x+y)}{x^{2} y^{2}}+\frac{1}{x y(1+x+y)}-\frac{\ln (1+x)}{x^{2} y^{2}}-\frac{\ln (1+y)}{x^{2} y^{2}} .
$$

We point out that this function is regular at the origin,

$$
\lim _{x, y \rightarrow 0} H(x, y)=\frac{1}{2} .
$$

Hence, the action (3.16) with this function is well-defined and the harmonic integral does not encounter any singularities.

The contributions from the last two terms in (3.20) to the action (3.16) vanish on shell due to the properties $(3.10)^{5}$. Therefore, the on-shell effective action can be rewritten in the following explicit form

$$
\Gamma=\frac{\alpha}{8} \int d \zeta\left(\begin{array}{l}
33 \\
11
\end{array}\right) d u\left[\frac{\left(c^{i} \bar{c}_{i}\right)^{2}}{c^{3} c^{3} \bar{c}_{1} \bar{c}_{1}} \ln \left(1+\frac{\bar{\omega}^{12} c^{3}}{c^{i} \bar{c}_{i}}+\frac{\omega_{23} \bar{c}_{1}}{c^{i} \bar{c}_{i}}\right)+\frac{\left(c^{i} \bar{c}_{i}\right) \bar{\omega}^{12} \omega_{23}}{c^{3} \bar{c}_{1}\left(c^{i} \bar{c}_{i}+\bar{\omega}^{12} c^{3}+\omega_{23} \bar{c}_{1}\right)}\right] .
$$

Although the charged objects $c^{3}$ and $\bar{c}_{1}$ appear in the denominators, they do not lead to the divergent harmonic integrals. It can be explicitly checked that upon passing to the component form of the action (3.22), all dangerous terms with divergent harmonic integrals vanish after performing the integration over the Grassmann variables. Some component terms of this action will be studied in the next Section.

\subsection{Complete $\mathcal{N}=3$ superconformal symmetry}

Now we consider the transformations (2.25) which include all parameters of the superconformal transformations. The corresponding variations (3.14) of the shifted superfield strengths $\bar{\omega}^{12}$ and $\omega_{23}$ read

$$
\begin{aligned}
& \delta_{\mathrm{sc}} \bar{\omega}^{12}=A \bar{\omega}^{12}+A \bar{c}_{3}+\lambda_{3}^{2} \bar{c}_{2}+\lambda_{3}^{1} \bar{c}_{1}, \\
& \delta_{\mathrm{sc}} \omega_{23}=\bar{A} \omega_{23}+\bar{A} c^{1}-\lambda_{2}^{1} c^{2}-\lambda_{3}^{1} c^{3},
\end{aligned}
$$

where $A$ and $\bar{A}$ are given in (2.26) and $\lambda_{J}^{I}$ are defined in (2.18). Under these transformations the action (3.16) varies as

$$
\begin{aligned}
\delta_{\mathrm{sc}} \Gamma= & \frac{\alpha}{8} \int d \zeta\left(\begin{array}{l}
33 \\
11
\end{array}\right) d u\left(\bar{\omega}^{12} \omega_{23}\right)^{2}\left[\frac{2}{x} H(x, y)+H_{x}^{\prime}(x, y)\right]\left[A x+A c^{3} \bar{c}_{3}+\lambda_{3}^{2} c^{3} \bar{c}_{2}+\lambda_{3}^{1} c^{3} \bar{c}_{1}\right] \\
& +\frac{\alpha}{8} \int d \zeta\left(\begin{array}{l}
33 \\
11
\end{array}\right) d u\left(\bar{\omega}^{12} \omega_{23}\right)^{2}\left[\frac{2}{y} H(x, y)+H_{y}^{\prime}(x, y)\right]\left[\bar{A} y+\bar{A} c^{1} \bar{c}_{1}-\lambda_{2}^{1} c^{2} \bar{c}_{1}-\lambda_{3}^{1} c^{3} \bar{c}_{1}\right] .
\end{aligned}
$$

\footnotetext{
${ }^{5}$ The properties (3.10) are valid essentially on shell. Therefore the last two terms in (3.20) can be neglected only on shell although they can be important for the off-shell completion of the action.
} 
For simplicity we set here $c^{i} \bar{c}_{i}=1$, so $x=\bar{\omega}^{12} c^{3}, y=\omega_{23} \bar{c}_{1}$. The first and second lines in (3.24) are complex-conjugated to each other.

Given the explicit form (3.20) of the function $H(x, y)$, it is easy to check that it solves the following differential equations

$$
\begin{aligned}
& \frac{2}{x} H(x, y)+H_{x}^{\prime}(x, y)=\frac{1}{x(1+x)(1+x+y)^{2}}, \\
& \frac{2}{y} H(x, y)+H_{y}^{\prime}(x, y)=\frac{1}{y(1+y)(1+x+y)^{2}} .
\end{aligned}
$$

Taking them into account, we are going to show that the integrand in (3.24) is a total harmonic derivative, so the variation (3.24) vanishes.

To this end, we introduce the auxiliary functions $f(x, y)$ and $\tilde{f}(x, y)$ :

$$
\begin{aligned}
& f(x, y)=\frac{1}{y(y+1)(x+y+1)}+\frac{\ln (1+x+y)}{x y^{2}}-\frac{\ln (1+x)}{x y^{2}}-\frac{\ln (1+y)}{x y^{2}}, \\
& \tilde{f}(x, y)=f(y, x)=\frac{1}{x(x+1)(x+y+1)}+\frac{\ln (1+x+y)}{y x^{2}}-\frac{\ln (1+y)}{y x^{2}}-\frac{\ln (1+x)}{y x^{2}} .
\end{aligned}
$$

They possess the following properties

$$
\begin{aligned}
x f_{x}^{\prime}+f & =-\frac{1}{(1+x)(1+x+y)^{2}}=-\left(x H_{x}^{\prime}+2 H\right), \\
x f_{x}^{\prime}+y f_{y}^{\prime}+3 f & =\frac{1}{x(1+x)(1+x+y)^{2}}-\frac{1}{x(1+y)^{2}}=\left(H_{x}^{\prime}+\frac{2}{x} H\right)+\ldots, \\
y \tilde{f}_{y}^{\prime}+\tilde{f} & =-\frac{1}{(1+y)(1+x+y)^{2}}=-\left(y H_{y}^{\prime}+2 H\right), \\
y \tilde{f}_{y}^{\prime}+x \tilde{f}_{x}^{\prime}+3 \tilde{f} & =\frac{1}{y(1+y)(1+x+y)^{2}}-\frac{1}{y(1+x)^{2}}=\left(H_{y}^{\prime}+\frac{2}{x} H\right)+\ldots .
\end{aligned}
$$

Here dots stand for the terms integrals of which over the analytic superspace with the weight $\left(\bar{\omega}^{12} \omega_{23}\right)^{2}$ are on-shell vanishing due to the relations (3.10). Up to these terms, the equations (3.27)-(3.30) allow one to deduce the following relations

$$
\begin{aligned}
-D_{3}^{2}\left(f(x, y) c^{3} \bar{c}_{2} A\right)-D_{3}^{1}\left(f(x, y) c^{3} \bar{c}_{1} A\right)= & \left(H_{x}^{\prime}+\frac{2}{x} H\right)\left(A x+A c^{3} \bar{c}_{3}\right) \\
& -f(x, y) c^{3} \bar{c}_{2} \lambda_{3}^{2}-f(x, y) c^{3} \bar{c}_{1} \lambda_{3}^{1}, \\
D_{2}^{1}\left(\tilde{f}(x, y) c^{2} \bar{c}_{1} \bar{A}\right)+D_{3}^{1}\left(\tilde{f}(x, y) c^{3} \bar{c}_{1} \bar{A}\right)= & \left(H_{y}^{\prime}+\frac{2}{y} H\right)\left(\bar{A} y+\bar{A} c^{1} \bar{c}_{1}\right) \\
& +\tilde{f}(x, y) c^{2} \bar{c}_{1} \lambda_{2}^{1}+\tilde{f}(x, y) c^{3} \bar{c}_{1} \lambda_{3}^{1} .
\end{aligned}
$$

Here we made use of the following simple identities

$$
\lambda_{2}^{1}=D_{2}^{1} \bar{A}, \quad \lambda_{3}^{2}=D_{3}^{2} A, \quad \lambda_{3}^{1}=D_{3}^{1} A=D_{3}^{1} \bar{A},
$$

as well as of the convention $c^{i} \bar{c}_{i}=1$.

Next, we introduce the functions

$$
\begin{aligned}
& g(x, y)=\frac{1}{y(1+y)^{2}(1+x+y)}-\frac{1}{y(x+1)}, \\
& \tilde{g}(x, y)=g(y, x)=\frac{1}{x(1+x)^{2}(1+x+y)}-\frac{1}{x(y+1)},
\end{aligned}
$$


with the properties

$$
\begin{aligned}
x g_{x}^{\prime}+g & =\frac{1}{y(1+y)(1+x+y)^{2}}-\frac{1}{y(1+x)^{2}}=H_{y}^{\prime}+\frac{2}{y} H+\ldots, \\
y \tilde{g}_{y}^{\prime}+\tilde{g} & =\frac{1}{x(1+x)(1+x+y)^{2}}-\frac{1}{x(1+y)^{2}}=H_{x}^{\prime}+\frac{2}{x} H+\ldots, \\
g(x, y)-\tilde{g}(x, y) & =\left(H_{x}^{\prime}+\frac{2}{x} H\right)-\left(H_{y}^{\prime}+\frac{2}{y} H\right) .
\end{aligned}
$$

Here, as in (3.28) and in (3.30), the dots stand for the terms vanishing on shell after integration over the analytic superspace with the weight $\left(\bar{\omega}^{12} \omega_{23}\right)^{2}$. Up to these terms, we obtain the following relation

$$
\begin{aligned}
-D_{2}^{1}\left(\lambda_{3}^{2} \tilde{g}(x, y) c^{3} \bar{c}_{1}\right)-D_{3}^{2}\left(\lambda_{2}^{1} g(x, y) c^{3} \bar{c}_{1}\right)= & \left(H_{x}^{\prime}+\frac{2}{x} H\right) \lambda_{3}^{2} c^{3} \bar{c}_{2}-\left(H_{y}^{\prime}+\frac{2}{y} H\right) \lambda_{2}^{1} c^{2} \bar{c}_{1} \\
& +\left[\left(H_{x}^{\prime}+\frac{2}{x} H\right)-\left(H_{y}^{\prime}+\frac{2}{y} H\right)\right] \lambda_{3}^{1} c^{3} \bar{c}_{1} .
\end{aligned}
$$

Finally, introduce the functions

$$
\begin{aligned}
& h(x, y)=-\frac{1}{(1+x) y}+\frac{\ln (1+x)}{x y^{2}}+\frac{\ln (1+y)}{x y^{2}}-\frac{\ln (1+x+y)}{x y^{2}} \\
& \tilde{h}(x, y)=h(y, x)=-\frac{1}{(1+y) x}+\frac{\ln (1+y)}{y x^{2}}+\frac{\ln (1+x)}{y x^{2}}-\frac{\ln (1+x+y)}{y x^{2}},
\end{aligned}
$$

with the properties

$$
\begin{gathered}
h(x, y)+y h_{y}^{\prime}(x, y)=f(x, y), \quad \tilde{h}(x, y)+x \tilde{h}_{x}^{\prime}(x, y)=\tilde{f}(x, y), \\
h-\tilde{h}=\tilde{f}-f .
\end{gathered}
$$

These properties allow one to derive the following relation

$$
-D_{2}^{1}\left(\lambda_{3}^{2} h(x, y) c^{3} \bar{c}_{1}\right)-D_{3}^{2}\left(\lambda_{2}^{1} \tilde{h}(x, y) c^{3} \bar{c}_{1}\right)=f \lambda_{3}^{2} c^{3} \bar{c}_{2}-\tilde{f} \lambda_{2}^{1} c^{2} \bar{c}_{1}+(f-\tilde{f}) \lambda_{3}^{1} c^{3} \bar{c}_{1} .
$$

Now we put together the relations (3.31), (3.38) and (3.43) and observe that the variation (3.24) can be represented as a linear combination of harmonic derivatives acting on the quantities which are expressed through the functions (3.27), (3.34) and (3.40),

$$
\begin{aligned}
\delta_{\mathrm{sc}} \Gamma= & \frac{\alpha}{8} \int d \zeta\left(\begin{array}{l}
33 \\
11
\end{array}\right) d u\left(\bar{\omega}^{12} \omega_{23}\right)^{2}\left\{D_{2}^{1}\left(\tilde{f} c^{2} \bar{c}_{1} \bar{A}\right)-D_{3}^{2}\left(f c^{3} \bar{c}_{2} A\right)+D_{3}^{1}\left(\tilde{f} c^{3} \bar{c}_{1} \bar{A}-f c^{3} \bar{c}_{1} A\right)\right. \\
& \left.-D_{2}^{1}\left[(\tilde{g}+h) \lambda_{3}^{2} c^{3} \bar{c}_{1}\right]-D_{3}^{2}\left[(g+\tilde{h}) \lambda_{2}^{1} c^{3} \bar{c}_{1}\right]\right\}
\end{aligned}
$$

The variation (3.44) vanishes as an integral of total harmonic derivative. This proves the invariance of the action (3.22) under the full $\mathrm{SU}(2,2 \mid 3)$ superconformal group ${ }^{6}$.

\footnotetext{
${ }^{6}$ Note that $(3.22)$ is $\mathrm{SU}(2,2 \mid 3)$ invariant for any $c^{i} \neq 0$, without any restriction on the norm $c^{i} \bar{c}_{i}$ which was put equal to 1 in the above consideration merely for convenience.
} 


\subsection{Independence of the choice of vacua}

By construction, the effective action (3.16) with the function (3.20) is meaningful only on the Coulomb branch of the $\mathcal{N}=3$ SYM theory. This is manifested in the explicit presence of non-zero vev constants $c^{i}$ and $\bar{c}_{i}$ in the Lagrangian in (3.16). However, the action itself should be independent of any particular choice of these constants, except for the point $c^{i}=0$ at which the effective action is singular.

Let us rewrite (3.16) in terms of the original (non-shifted) superfield strengths $\bar{W}^{12}$ and $W_{23}$

$$
\Gamma\left[\bar{W}^{12}, W_{23} ; c^{i}, \bar{c}_{i}\right]=\frac{\alpha}{8} \int d \zeta\left(\begin{array}{l}
33 \\
11
\end{array}\right) d u \frac{\left(\bar{W}^{12}-\bar{c}_{3}\right)^{2}\left(W_{23}-c^{1}\right)^{2}}{\left(c^{i} \bar{c}_{i}\right)^{2}} H\left(c^{3} \frac{\bar{W}^{12}-\bar{c}_{3}}{c^{i} \bar{c}_{i}}, \bar{c}_{1} \frac{W_{23}-c^{1}}{c^{i} \bar{c}_{i}}\right) .
$$

In the previous subsection we proved that this action is invariant under the full $\mathrm{SU}(2,2 \mid 3) \mathrm{su}-$ perconformal group. Taking into account that the analytic integration measure is $\mathrm{SU}(2,2 \mid 3)$ invariant by itself, the property of superconformal invariance of the action can be written in the finite form as

$$
\Gamma\left[\bar{W}^{12}, W_{23} ; c^{i}, \bar{c}_{i}\right]=\Gamma^{\prime}\left[\bar{W}^{12 \prime}, W_{23}{ }^{\prime} ; c^{i}, \bar{c}_{i}\right]=\Gamma\left[\bar{W}^{12 \prime}, W_{23}{ }^{\prime} ; c^{i}, \bar{c}_{i}\right] .
$$

In particular, consider scale and $\gamma_{5}$ transformations of the superfield strength in the finite form,

$$
\bar{W}^{12} \rightarrow e^{\bar{A}} \bar{W}^{12}, \quad W_{23} \rightarrow e^{A} W_{23},
$$

where $A=-a+2 i b$. The transformation of the action (3.45) under (3.47) can be represented as

$$
\begin{aligned}
\Gamma\left[\bar{W}^{12}, W_{23} ; c^{i}, \bar{c}_{i}\right]= & \Gamma\left[e^{\bar{A}} \bar{W}^{12}, e^{A} W_{23} ; c^{i}, \bar{c}_{i}\right] \\
= & \frac{\alpha}{8} \int d \zeta\left(\begin{array}{l}
33 \\
11
\end{array}\right) d u \frac{\left(\bar{W}^{12}-e^{-\bar{A}} \bar{c}_{3}\right)^{2}\left(W_{23}-e^{-A} c^{1}\right)^{2}}{\left(e^{-A-\bar{A}} c^{i} \bar{c}_{i}\right)^{2}} \\
& \times H\left(e^{-A} c^{3} \frac{\bar{W}^{12}-e^{-\bar{A}} \bar{c}_{3}}{e^{-A-\bar{A}} c^{i} \bar{c}_{i}}, e^{-\bar{A}} \bar{c}_{1} \frac{W_{23}-e^{-A} c^{1}}{e^{-A-\bar{A}} c^{i} \bar{c}_{i}}\right) .
\end{aligned}
$$

So, all $A$-dependence is absorbed into the vev constants, $c^{i} \rightarrow e^{-A} c^{i}, \bar{c}_{i} \rightarrow e^{-\bar{A}} \bar{c}_{i}$. Hence, the superconformal invariance of the action (3.45) implies its independence of complex rescalings of the vev constants,

$$
\Gamma\left[\bar{W}^{12}, W_{23} ; c^{i}, \bar{c}_{i}\right]=\Gamma\left[e^{\bar{A}} \bar{W}^{12}, e^{A} W_{23} ; c^{i}, \bar{c}_{i}\right]=\Gamma\left[\bar{W}^{12}, W_{23} ; e^{-\bar{A}} c^{i}, e^{-A} \bar{c}_{i}\right]
$$

In a similar way, one can prove that the action (3.45) is independent of the parameters of finite $\mathrm{SU}(3)$ rotations of the vev constants,

$$
\Gamma\left[\bar{W}^{12}, W_{23} ; c^{i}, \bar{c}_{i}\right]=\Gamma\left[\bar{W}^{12}, W_{23} ; \Lambda_{j}^{i} c^{j}, \bar{\Lambda}_{i}^{j} \bar{c}_{j}\right]
$$

where $\Lambda_{j}^{i}$ are $\mathrm{SU}(3)$ matrices. As a result, the action (3.45) is independent of any particular choice of the vacuum $c^{i}, c^{i} \neq 0$. 
Perhaps, it make sense to give a more detailed proof of the latter statement. It goes as follows. Let us assume, without loss of generality, that $c^{3} \neq 0$. Then, using the coset $\mathrm{SU}(3) /[\mathrm{U}(1) \times \mathrm{SU}(2)]$ transformations with a constant $\mathrm{SU}(2)$ doublet as parameters, one can cast $c^{i}$ in the form $c^{i}=\left(0,0, c^{3}\right)$. The constant $c^{3}$ can be made real by making use of the residual $\mathrm{U}(1)$ transformation (a combination of the $\gamma_{5}$ transformations and those of $\mathrm{U}(1)$ from the denominator of $\mathrm{SU}(3) /[\mathrm{U}(1) \times \mathrm{SU}(2)])$. Finally, it can be rescaled to any non-zero value, keeping in mind the independence of the action of the rescalings of the vev constants.

\section{Component structure}

\section{1 $F^{4} / X^{4}$ term}

To derive this term from the effective action (3.16), it is sufficient to consider only constant Maxwell and scalar fields, omitting all other components in (2.11),

$$
\hat{\bar{\omega}}^{12}=u_{i}^{1} \phi^{i}+4 i \theta_{2}^{\alpha} \theta_{3}^{\beta} F_{\alpha \beta}, \quad \hat{\omega}_{23}=\bar{u}_{3}^{i} \bar{\phi}_{i}+4 i \bar{\theta}^{1 \dot{\alpha}} \bar{\theta}^{2 \dot{\beta}} \bar{F}_{\dot{\alpha} \dot{\beta}} .
$$

Substituting these superfields into (3.16), we integrate over the Grassmann variables to obtain

$$
\Gamma_{F^{4} / X^{4}}=\frac{\alpha}{2} \int d^{4} x d u F^{2} \bar{F}^{2} \sum_{m, n=0}^{\infty} \frac{(m+1)(n+1)(m+n+2) !(-1)^{m+n}}{m ! n !}\left(\bar{\phi}_{3} c^{3}\right)^{m}\left(\phi^{1} \bar{c}_{1}\right)^{n} .
$$

Here we used the series expansion (3.17) for the function $H$ with the coefficients given by (3.19). In this subsection we assume $c^{i} \bar{c}_{i}=1$ for simplicity and use the notation $F^{2}=F^{\alpha \beta} F_{\alpha \beta}, \bar{F}^{2}=\bar{F}^{\dot{\alpha} \dot{\beta}} \bar{F}_{\dot{\alpha} \dot{\beta}}$.

It is convenient to represent (4.2) as a sum of two terms,

$$
\Gamma_{F^{4} / X^{4}}=\frac{\alpha}{2} \int d^{4} x F^{2} \bar{F}^{2}\left(T_{1}+T_{2}\right)
$$

where

$$
\begin{aligned}
& T_{1}=\int d u \sum_{n=0}^{\infty} \sum_{m=0}^{n} \frac{(m+1)(n+1)(m+n+2) !(-1)^{m+n}}{m ! n !}\left(\phi^{1} \bar{c}_{1}\right)^{n}\left(\bar{\phi}_{3} c^{3}\right)^{m} \\
& T_{2}=\int d u \sum_{n=0}^{\infty} \sum_{m=n+1}^{\infty} \frac{(m+1)(n+1)(m+n+2) !(-1)^{m+n}}{m ! n !}\left(\phi^{1} \bar{c}_{1}\right)^{n}\left(\bar{\phi}_{3} c^{3}\right)^{m} .
\end{aligned}
$$

The reason for this separation is that $m \leq n$ in $T_{1}$ while $m>n$ in $T_{2}$. Therefore, for each of these terms we can apply the equation (B.5) for the harmonic integrals,

$$
\begin{aligned}
& T_{1}=2 \sum_{n=0}^{\infty} \sum_{m=0}^{n} \sum_{l=0}^{m} \frac{(m+n-l+1) !(-1)^{m+n+l}}{l !(n-l) !(m-l) !}\left(\phi^{i} \bar{\phi}_{i}\right)^{l}\left(\phi^{i} \bar{c}_{i}\right)^{n-l}\left(c^{i} \bar{\phi}_{i}\right)^{m-l}, \\
& T_{2}=2 \sum_{n=0}^{\infty} \sum_{m=n+1}^{\infty} \sum_{l=0}^{n} \frac{(m+n-l+1) !(-1)^{m+n+l}}{l !(n-l) !(m-l) !}\left(\phi^{i} \bar{\phi}_{i}\right)^{l}\left(\phi^{i} \bar{c}_{i}\right)^{n-l}\left(c^{i} \bar{\phi}_{i}\right)^{m-l} .
\end{aligned}
$$


Changing the order of summation, these terms can be rewritten as

$$
\begin{aligned}
& T_{1}=2 \sum_{l, m=0}^{\infty} \sum_{n=m}^{\infty} \frac{(n+m+l+1) !(-1)^{m+n+l}}{l ! m ! n !}\left(\phi^{i} \bar{\phi}_{i}\right)^{l}\left(\phi^{i} \bar{\phi}_{i}\right)^{n}\left(c^{i} \bar{\phi}_{i}\right)^{m}, \\
& T_{2}=2 \sum_{l, m=0}^{\infty} \sum_{n=0}^{m-1} \frac{(n+m+l+1) !(-1)^{m+n+l}}{l ! m ! n !}\left(\phi^{i} \bar{\phi}_{i}\right)^{l}\left(\phi^{i} \bar{\phi}_{i}\right)^{n}\left(c^{i} \bar{\phi}_{i}\right)^{m} .
\end{aligned}
$$

Putting these two expressions together, we find

$$
\begin{aligned}
T_{1}+T_{2} & =2 \sum_{m, n, k=0}^{\infty} \frac{(-1)^{m+n+k}(m+n+k+1) !}{m ! n ! k !}\left(c^{i} \bar{\phi}_{i}\right)^{m}\left(\bar{c}_{i} \phi^{i}\right)^{n}\left(\bar{\phi}_{i} \phi^{i}\right)^{k} \\
& =\frac{2}{\left(1+c^{i} \bar{\phi}_{i}+\bar{c}_{i} \phi^{i}+\phi^{i} \bar{\phi}_{i}\right)^{2}}=\frac{2}{\left(\varphi^{i} \bar{\varphi}_{i}\right)^{2}} .
\end{aligned}
$$

As a result, the $F^{4} / X^{4}$ term in the effective action reads

$$
\Gamma_{F^{4} / X^{4}}=\alpha \int d^{4} x \frac{F^{2} \bar{F}^{2}}{\left(\varphi^{i} \bar{\varphi}_{i}\right)^{2}} .
$$

This expression is explicitly scale and $\mathrm{U}(3)$ invariant, as is expected.

It is a highly non-trivial and remarkable phenomenon that the vev constants $c^{i}$ and the shifted scalars $\phi^{i}$ have combined into the initial scalar fields $\varphi^{i},(3.11)$, after doing the Grassmann and harmonic integrals which is a rather involved procedure in its own. This confirms the independence of the action (3.16) of any particular choice of the vacua, the fact that was proved in the previous section.

Note that (4.8) also respects hidden $\mathrm{SO}(6) \simeq \mathrm{SU}(4)$ invariance, with the $\mathrm{SU}(4) / \mathrm{U}(3)$ transformations acting as

$$
\delta \varphi^{i}=\varepsilon^{i k l} \lambda_{k} \bar{\varphi}_{l}, \quad \delta \bar{\varphi}_{i}=\varepsilon_{i k l} \bar{\lambda}^{k} \varphi^{l},
$$

where $\lambda_{i}$ comprise 6 corresponding group parameters. This is an indication that the superfield effective action (3.16), besides the superconformal $\mathrm{SU}(2,2 \mid 3)$ symmetry, enjoys as well an on-shell SU(4) symmetry, and hence, the superconformal SU(2,2|4) symmetry as a closure of the two former ones. It would be interesting to explicitly find the realization of this $\mathrm{SU}(4)$ on the analytic superfield strengths.

\section{$4.2 \quad F^{6} / X^{8}$ term}

It is known that the non-conformal action (3.1) produces not only the $F^{4}$ term but also the $F^{6}$ term in the Born-Infeld action [6]. The $F^{6}$ term appears essentially on shell, when some of the auxiliary fields are eliminated by their effective equations of motion. In [7] it was conjectured that this procedure should work in a similar way in the superconformal case, with the $F^{6} / X^{8}$ term as the outcome. Here we show that this is indeed the case and present details of the relevant derivation.

As shown in [6], the Maxwell field strength $F_{m n}=\partial_{m} A_{n}-\partial_{n} A_{m}$ is accompanied by the antisymmetric tensor auxiliary field $H_{m n}=-H_{n m}$. When the on-shell constraints 
are relaxed, these fields appear in the superfield strengths in the combination $V_{m n}=$ $\frac{1}{4}\left(F_{m n}+H_{m n}\right)$,

$$
\bar{\omega}^{12}=u_{i}^{1} \phi^{i}+4 i \theta_{2}^{\alpha} \theta_{3}^{\beta} V_{\alpha \beta}+\ldots, \quad \omega_{23}=\bar{u}_{3}^{i} \bar{\phi}_{i}+4 i \bar{\theta}^{1 \dot{\alpha}} \bar{\theta}^{2 \dot{\beta}} \bar{V}_{\dot{\alpha} \dot{\beta}}+\ldots
$$

Here $V_{\alpha \beta}$ and $\bar{V}_{\dot{\alpha} \dot{\beta}}$ are spinorial components of the antisymmetric tensor $V_{m n}$ and dots stand for the other field components which are irrelevant for our consideration. The part of the free classical action $S_{2}$ which involves these fields reads [6]

$$
S_{2}=\int d^{4} x\left[V^{2}+\bar{V}^{2}-2(V F+\bar{V} \bar{F})+\frac{1}{2}\left(F^{2}+\bar{F}^{2}\right)\right] .
$$

One can recover the standard Maxwell action for $F_{m n}$ upon eliminating the auxiliary fields from $S_{2}$. However, our purpose is to eliminate them from the effective equations of motion, when the action (3.16) is added to the classical free SYM action. The superfield effective equations of motion are derived in Appendix D. Here we need only some SU(3) singlet sub-sector of the component expansion of these equations, so it is simpler to derive it independently.

As in the previous subsection, we substitute the superfields (4.10) into (3.16) and find

$$
\Gamma_{F^{4} / X^{4}}=\alpha \int d^{4} x \frac{V^{2} \bar{V}^{2}}{\left(\varphi^{i} \bar{\varphi}_{i}\right)^{2}} .
$$

The action $S_{2}+\Gamma_{F^{4} / X^{4}}$ produces the following equations of motion for the auxiliary fields $V_{\alpha \beta}$ and $\bar{V}_{\dot{\alpha} \dot{\beta}}$,

$$
F_{\alpha \beta}=V_{\alpha \beta}\left[1+\alpha \frac{\bar{V}^{2}}{\left(\varphi^{i} \bar{\varphi}_{i}\right)^{2}}\right], \quad \bar{F}_{\dot{\alpha} \dot{\beta}}=\bar{V}_{\dot{\alpha} \dot{\beta}}\left[1+\alpha \frac{V^{2}}{\left(\varphi^{i} \bar{\varphi}_{i}\right)^{2}}\right] .
$$

The solution of these equations can be represented as a series in the Maxwell field strength, in which we need only the lowest terms,

$$
V_{\alpha \beta}=F_{\alpha \beta}\left[1-\alpha \frac{\bar{F}^{2}}{\left(\varphi^{i} \bar{\varphi}_{i}\right)^{2}}+O\left(F^{3}\right)\right], \quad \bar{V}_{\dot{\alpha} \dot{\beta}}=\bar{F}_{\dot{\alpha} \dot{\beta}}\left[1-\alpha \frac{F^{2}}{\left(\varphi^{i} \bar{\varphi}_{i}\right)^{2}}+O\left(F^{3}\right)\right] .
$$

Substituting these solutions back into $S_{2}+\Gamma_{F^{4} / X^{4}}$, we earn the correct $F^{6}$ term,

$$
S_{2}+\Gamma_{F^{4} / X^{4}}=\int d^{4} x\left[-\frac{1}{2}\left(F^{2}+\bar{F}^{2}\right)+\alpha \frac{F^{2} \bar{F}^{2}}{\left(\varphi^{i} \bar{\varphi}_{i}\right)^{2}}-\alpha^{2} \frac{F^{2} \bar{F}^{2}}{\left(\varphi^{i} \bar{\varphi}_{i}\right)^{2}}\left(F^{2}+\bar{F}^{2}\right)+O\left(F^{8}\right)\right] .
$$

With $\alpha=Q / 2$ this action coincides, up to the $F^{6}$ order, with the Born-Infeld part of the effective worldvolume action for a D3 brane moving in curved $A d S_{5} \times S^{5}$ vacuum background of type IIB supergravity $[28]^{7}$,

$$
\begin{aligned}
& \int d^{4} x \frac{|X|^{4}}{Q}\left[1-\sqrt{-\operatorname{det}\left(\eta_{m n}+Q^{1 / 2}|X|^{-2} F_{m n}\right)}\right] \\
= & -\frac{1}{2} \int d^{4} x\left[F^{2}+\bar{F}^{2}-\frac{Q}{|X|^{4}} F^{2} \bar{F}^{2}+\frac{1}{2} \frac{Q^{2}}{|X|^{8}} F^{2} \bar{F}^{2}\left(F^{2}+\bar{F}^{2}\right)+O\left(F^{8}\right)\right] .
\end{aligned}
$$

\footnotetext{
${ }^{7}$ We omit here all terms with derivatives of scalars $X^{I}$.
} 
In stringy language, we can make the identification $Q=N g_{s} \alpha^{2} / \pi$, where $N$ is the number of D3 branes which induce the $A d S_{5} \times S^{5}$ geometry, $g_{s}$ is the string coupling and $\alpha^{\prime}$ is the inverse string tension. It was conjectured in [29-33] (see also [34] for a review) that such D3 brane effective action should coincide with the low-energy effective action of $\mathcal{N}=4 \mathrm{SU}(N)$ SYM theory in the large $N$ limit. Thus here we proved this conjecture up to the $F^{6} / X^{8}$ order.

\subsection{A comment on the Wess-Zumino term}

As shown in $[22,23]$, the quantum effective action of $\mathcal{N}=4$ SYM theory must contain a Wess-Zumino (WZ) type non-tensor term in the scalar fields sector. The presence of such term in various superfield versions of the $\mathcal{N}=4$ SYM effective action (in particular, in its $\mathcal{N}=2$ superfield version [17]) was recently proved in [35, 36]. Here we give an evidence that the $\mathcal{N}=3$ effective action (3.16) also contains WZ term in its component expansion.

To detect the WZ term, it is sufficient to keep only scalar fields in the superfields $(2.11)$

$$
\begin{aligned}
& \hat{\omega}_{23}=\phi^{1}+2 i \theta_{2}^{\alpha} \bar{\theta}^{2 \dot{\alpha}} \partial_{\alpha \dot{\alpha}} \phi^{1}-4 i \theta_{2}^{\alpha} \bar{\theta}^{1 \dot{\alpha}} \partial_{\alpha \dot{\alpha}} \phi^{2}-4 i \theta_{3}^{\alpha} \bar{\theta}^{1 \dot{\alpha}} \partial_{\alpha \dot{\alpha}} \phi^{3}+8 \theta_{2}^{\alpha} \theta_{3}^{\beta} \bar{\theta}^{1 \dot{\alpha}} \bar{\theta}^{2 \dot{\beta}} \partial_{\alpha \dot{\alpha}} \partial_{\beta \dot{\beta}} \phi^{3}, \\
& \hat{\bar{\omega}}^{12}=\bar{\phi}_{3}-2 i \theta_{2}^{\alpha} \bar{\theta}^{2 \dot{\alpha}} \partial_{\alpha \dot{\alpha}} \bar{\phi}_{3}+4 i \theta_{3}^{\alpha} \bar{\theta}^{1 \dot{\alpha}} \partial_{\alpha \dot{\alpha}} \bar{\phi}_{1}+4 i \theta_{3}^{\alpha} \bar{\theta}^{2 \dot{\alpha}} \partial_{\alpha \dot{\alpha}} \bar{\phi}_{2}+8 \bar{\theta}^{1 \dot{\alpha}} \bar{\theta}^{2 \dot{\beta}} \theta_{2}^{\alpha} \theta_{3}^{\beta} \partial_{\alpha \dot{\alpha}} \partial_{\beta \dot{\beta}} \bar{\varphi}_{1} .
\end{aligned}
$$

We substitute these superfields into the action (3.16) and integrate over the Grassmann variables, keeping only those terms which contain four derivatives contracted with the antisymmetric $\varepsilon$-symbol,

$$
\begin{aligned}
\Gamma_{W Z}= & -\frac{i \alpha}{8} \varepsilon^{m n p q} \int d^{4} x d u\left[\partial_{m} \phi^{2} \partial_{n} \bar{\phi}_{3} \partial_{p} \bar{\phi}_{2} \partial_{q} \phi^{3}+\partial_{m} \bar{\phi}_{2} \partial_{n} \bar{\phi}_{1} \partial_{p} \phi^{2} \partial_{q} \phi^{1}\right] \\
& \times \sum_{i, j=0}^{\infty}(-1)^{i+j} \frac{(i+j+2) !(i+1)(j+1)}{i ! j !}\left(c^{3} \bar{\phi}_{3}\right)^{i}\left(\bar{c}_{1} \phi^{1}\right)^{j}
\end{aligned}
$$

To compare this expression with the standard expression (C.1) for WZ term ${ }^{8}$, it is necessary to compute the harmonic integrals and to sum the series. Unfortunately, it is very difficult to find the explicit expression for the integral

$$
\int d u u_{i_{1}}^{1} \bar{u}_{1}^{i_{1}^{\prime}} \ldots u_{i_{n}}^{1} \bar{u}_{1}^{i_{n}^{\prime}} u_{j_{1}}^{3} \bar{u}_{3}^{j_{1}^{\prime}} \ldots u_{j_{m}}^{3} \bar{u}_{3}^{j_{m}^{\prime}} u_{k}^{2} \bar{u}_{2}^{k^{\prime}}
$$

in terms of (anti)symmetrized irreducible combinations of the delta-symbols. Therefore here we restrict ourselves to considering only the lowest terms in (4.18), namely,

$\Gamma_{W Z}=\frac{3}{2} i \alpha \varepsilon^{m n p q} \int d^{4} x d u\left[\partial_{m} \phi^{2} \partial_{n} \bar{\phi}_{3} \partial_{p} \bar{\phi}_{2} \partial_{q} \phi^{3}+\partial_{m} \bar{\phi}_{2} \partial_{n} \bar{\phi}_{1} \partial_{p} \phi^{2} \partial_{q} \phi^{1}\right]\left(c^{3} \bar{\phi}_{3}+\bar{c}_{1} \phi^{1}\right)+O\left(\phi^{6}\right)$.

The corresponding harmonic integral is quite easy to do,

$$
\int d u u_{i}^{1} u_{j}^{2} u_{k}^{3} \bar{u}_{1}^{i^{\prime}} \bar{u}_{2}^{j^{\prime}} \bar{u}_{3}^{k^{\prime}}=\frac{1}{36} \varepsilon_{i j k} \varepsilon^{i^{\prime} j^{\prime} k^{\prime}}+\frac{1}{60} \delta_{i}^{\left(i^{\prime}\right.} \delta_{j}^{j^{\prime}} \delta_{k}^{\left.k^{\prime}\right)}+\frac{1}{18} \delta_{i}^{\left(i^{\prime}\right.} \delta_{j}^{\left[j^{\prime}\right)} \delta_{k}^{\left.k^{\prime}\right]}+\frac{1}{18} \delta_{i}^{\left[i^{\prime}\right.} \delta_{j}^{\left(j^{\prime}\right]} \delta_{k}^{\left.k^{\prime}\right)} .
$$

\footnotetext{
${ }^{8}$ To be precise, we compare (4.18) with the WZ action in the four-dimensional form (C.19).
} 
Then it is straightforward to see that only the first term in the r.h.s. of (4.21) contributes to (4.20), while all other terms either vanish after contracting the indices or form total derivatives. As a result, (4.20) can be rewritten as

$$
\begin{aligned}
\Gamma_{W Z}=\frac{i \alpha}{24} \varepsilon^{m n p q} & \int d^{4} x \varepsilon_{i j k} \varepsilon^{i^{\prime} j^{\prime} k^{\prime}}\left[c^{i} \partial_{m} \phi^{j} \partial_{n} \phi^{k} \bar{\phi}_{i^{\prime}} \partial_{p} \bar{\phi}_{j^{\prime}} \partial_{q} \bar{\phi}_{k^{\prime}}\right. \\
& \left.-\phi^{i} \partial_{m} \phi^{j} \partial_{n} \phi^{k} \bar{c}_{i^{\prime}} \partial_{p} \bar{\phi}_{j^{\prime}} \partial_{q} \bar{\phi}_{k^{\prime}}\right]+O\left(\phi^{6}\right) .
\end{aligned}
$$

This expression coincides with (C.23) under the choice

$$
\alpha=-\frac{1}{2 \pi^{2}} .
$$

This proves that the action (3.16) contains the Wess-Zumino term. One of the possible four-dimensional representations of this term is given by the expression (C.19).

\section{Summary and discussion}

In the present paper we made an essential step towards solving the long-standing problem of constructing $\mathcal{N}=3 \mathrm{SYM}$ low-energy effective action in terms of unconstrained $\mathcal{N}=3$ superfields. We constructed the leading part of this effective action which is responsible for the $F^{4} / X^{4}$ term in components. This action is given by a local functional in the $\mathcal{N}=3$ analytic superspace, such that it depends on the $\mathcal{N}=3$ superfield strength without derivatives on them. The form of this functional is uniquely fixed by the requirements of scale and $\gamma_{5}$ invariance, although the action respects further $\mathrm{SU}(2,2 \mid 3)$ superconformal symmetry (and, perhaps, $\mathrm{SU}(2,2 \mid 4)$ ).

Since the $\mathcal{N}=3$ and $\mathcal{N}=4$ SYM models are equivalent on shell, the action (3.16) provides us with an $\mathcal{N}=3$ superfield description of the $\mathcal{N}=4$ SYM low-energy effective action. This effective action was previously studied in the $\mathcal{N}=2$ harmonic superspace $[17,18]$ and was rewritten in terms of the on-shell $\mathcal{N}=4$ superfields in $[35,36,38]$. In contrast to the representations of this action in the $\mathcal{N}=2$ and $\mathcal{N}=4$ harmonic superspaces, the Lagrangian in (3.16) has an explicit dependence on the vev constants $c^{i}=\left\langle\varphi^{i}\right\rangle$. However, this dependence is rather spurious: we proved that the action itself is in fact independent of any particular choice of these constants. This phenomenon is very similar to what one observed in the action of the $\mathcal{N}=2$ improved tensor multiplet [37] in the harmonic superspace [21] which also explicitly included the vev constants of the scalars, but this dependence disappeared in the full component action. In [21] it was argued that the presence of such constants reflects the non-trivial topological properties of this action. In our case the $\mathcal{N}=4 \mathrm{SYM}$ low-energy effective action also contains some topological term given by the WZ action for the scalar fields [22, 23]. Therefore the action (3.16) can be equally considered as an $\mathcal{N}=3$ superfield extension of the WZ term. A possible form of the WZ term arising from the $\mathcal{N}=3$ harmonic superspace is discussed in Appendix C, see eq. (C.19). The constants $c^{i}$ in this action break the manifest SU(3) symmetry, though the action is still $\mathrm{SU}(3)$ and $\mathrm{SU}(4)$ invariant up to total derivatives. This confirms the conclusions of [35] that the four-dimensional WZ term cannot be made manifestly invariant under $\mathrm{SU}(3)$ since this group is anomalous. 
In the present paper we studied the bosonic component structure of the action (3.16) in the limit of constant Maxwell and scalar fields and argued that it contains the WessZumino term. We showed that this action correctly reproduces the coefficients in front of the $F^{4} / X^{4}$ and $F^{6} / X^{8}$ terms to ensure their coincidence with the similar terms in the worldvolume action of D3 brane in the $A d S_{5} \times S^{5}$ background. To make the comparison of the action (3.16) with the D3 brane action more precise, it is necessary to study the component structure of (3.16) in the scalar field sector in more detail, beyond the constant field approximation. This problem is technically involved and will be addressed elsewhere.

Finally, it is worth pointing out that the action (3.16) was derived solely by employing the group-theory requirements of gauge invariance and superconformal symmetry. It is very desirable to develop the background field method for the $\mathcal{N}=3$ SYM theory in order to re-derive the action (3.16) from the quantum perturbation theory in $\mathcal{N}=3$ harmonic superspace $^{9}$. Note that the free propagators in the $\mathcal{N}=3$ harmonic superspace were studied in [5]. These methods might help to unveil the structure of effective action in the $\mathcal{N}=3$ and $\mathcal{N}=4 \mathrm{SYM}$ models beyond the low-energy approximation.

\section{Acknowledgments}

I.B.S. is indebted to D. Belyaev, S. Kuzenko, W. Schulgin and D. Sorokin for useful discussions. I.L.B. is grateful to CAPES for supporting his visit to the Physics Department of Universidade Federal de Juiz de Fora where the final part of work was done. The authors are grateful to the RFBR grant Nr. 11-02-90445 for partial support. I.L.B. and I.B.S. acknowledge the support from the RFBR grant Nr. 12-02-00121 and from LRSS grant Nr. 224.2012.2. The work of I.B.S. was also supported by the Marie Curie research fellowship Nr. 236231, "QuantumSupersymmetry". E.A.I. and B.M.Z. acknowledge the support from the RFBR grant Nr. 09-02-01209 and a grant of Heisenberg-Landau Program. E.A.I. thanks the Directorate of SUBATECH, University of Nantes, for the kind hospitality at the final stage of this work.

\section{A Derivation of scale and $\gamma_{5}$ invariant effective action}

Here we derive the equations for the coefficients $\alpha_{m, n}$ which follow from the requirement that the action (3.18) is invariant under (3.14). Consider two lowest terms in the series $(3.18)^{10}$,

$$
\begin{aligned}
& \Gamma_{0}=\alpha_{0,0} \int d \zeta\left(\begin{array}{l}
33 \\
11
\end{array}\right) d u\left(\bar{\omega}^{12} \omega_{23}\right)^{2}, \\
& \Gamma_{1}=\alpha_{0,1} \int d \zeta\left(\begin{array}{l}
33 \\
11
\end{array}\right) d u\left(\bar{\omega}^{12} \omega_{23}\right)^{2}\left(\bar{\omega}^{12} c^{3}+\omega_{23} \bar{c}_{1}\right) .
\end{aligned}
$$

\footnotetext{
${ }^{9}$ Like as the $\mathcal{N}=2$ superfield effective action of ref. [17] was re-derived from the $\mathcal{N}=2$ harmonic superfield perturbation theory in [18].

${ }^{10}$ Here, for simplicity, we put $c^{i} \bar{c}_{i}=1$ and $\alpha=32$.
} 
The superconformal variation of $\Gamma_{0}$ reads

$$
\delta_{\mathrm{sc}} \Gamma_{0}=2 \alpha_{0,0}(A+\bar{A}) \int d \zeta\left(\begin{array}{l}
33 \\
11
\end{array}\right) d u\left(\bar{\omega}^{12} \omega_{23}\right)^{2} .
$$

Note that the terms with $\bar{\omega}^{12} \bar{\omega}^{12} \omega_{23}$ and $\bar{\omega}^{12} \omega_{23} \omega_{23}$ vanish on shell because of the relations (3.10).

The superconformal variation of $\Gamma_{1}$ reads

$$
\delta_{\mathrm{sc}} \Gamma_{1}=3 \alpha_{0,1} \int d \zeta\left(\begin{array}{l}
33 \\
11
\end{array}\right) d u\left[\left(\bar{\omega}^{12} \omega_{23}\right)^{2}\left(\bar{A} c^{3} \bar{c}_{3}+A c^{1} \bar{c}_{1}\right)+O\left(\omega^{5}\right)\right] .
$$

Using the identities

$$
c^{1}=D_{2}^{1} c^{2}=D_{3}^{1} c^{3}, \quad \bar{c}_{3}=-D_{3}^{1} \bar{c}_{1}=-D_{3}^{2} \bar{c}_{2},
$$

which follow from the definitions (3.12), one can write

$$
\begin{aligned}
c^{1} \bar{c}_{1} & =\frac{1}{3}\left(c^{1} \bar{c}_{1}+\bar{c}_{1} D_{2}^{1} c^{2}+\bar{c}_{1} D_{3}^{1} c^{3}\right), \\
c^{3} \bar{c}_{3} & =\frac{1}{3}\left(c^{3} \bar{c}_{3}-c^{3} D_{3}^{1} \bar{c}_{1}-c^{3} D_{3}^{2} \bar{c}_{2}\right) .
\end{aligned}
$$

We substitute these expressions into (A.3) and integrate by parts with respect to the harmonic derivatives $D_{2}^{1}, D_{3}^{2}$ and $D_{3}^{1}$,

$$
\delta_{\mathrm{sc}} \Gamma_{1}=\alpha_{0,1} \int d \zeta\left(\begin{array}{l}
33 \\
11
\end{array}\right) d u\left[(\bar{A}+A)\left(\bar{\omega}^{12} \omega_{23}\right)^{2}+O\left(\omega^{5}\right)\right] .
$$

Here we made also use of the identity $c^{1} \bar{c}_{1}+c^{2} \bar{c}_{2}+c^{3} \bar{c}_{3}=c^{i} \bar{c}_{i}=1$. Comparing (A.6) with (A.2), we observe that the terms with four superfield strengths are canceled out under the condition

$$
\alpha_{0,1}=-2 \alpha_{0,0}
$$

Let us now consider the $n$-th term in the series (3.18),

$$
\Gamma_{n}=\int d \zeta\left(\begin{array}{l}
33 \\
11
\end{array}\right) d u\left(\bar{\omega}^{12} \omega_{23}\right)^{2} \sum_{i=0}^{n} \alpha_{i, n-i}\left(\bar{\omega}^{12} c^{3}\right)^{i}\left(\omega_{23} \bar{c}_{1}\right)^{n-i}
$$

and compute its variation under (3.14),

$$
\begin{aligned}
\delta_{\mathrm{sc}} \Gamma_{n}= & \int d \zeta\left(\begin{array}{l}
33 \\
11
\end{array}\right) d u\left(\bar{\omega}^{12} \omega_{23}\right)^{2} \sum_{i=0}^{n} \alpha_{i, n-i}[(i+2) \bar{A}+(n-i+2) A]\left(\bar{\omega}^{12} c^{3}\right)^{i}\left(\omega_{23} \bar{c}_{1}\right)^{n-i} \\
& +\int d \zeta\left(\begin{array}{l}
33 \\
11
\end{array}\right) d u\left(\bar{\omega}^{12} \omega_{23}\right)^{2} \sum_{i=1}^{n} \alpha_{i, n-i}(i+2) \bar{A}\left(\bar{\omega}^{12} c^{3}\right)^{i-1}\left(\omega_{23} \bar{c}_{1}\right)^{n-i} c^{3} \bar{c}_{3} \\
& +\int d \zeta\left(\begin{array}{l}
33 \\
11
\end{array}\right) d u\left(\bar{\omega}^{12} \omega_{23}\right)^{2} \sum_{i=0}^{n-1} \alpha_{i, n-i}(n-i+2) A\left(\bar{\omega}^{12} c^{3}\right)^{i}\left(\omega_{23} \bar{c}_{1}\right)^{n-i-1} c^{1} \bar{c}_{1} .
\end{aligned}
$$

In the second line of (A.9) we apply the identity

$$
\bar{c}_{3}\left(c^{3}\right)^{i}\left(\bar{c}_{1}\right)^{n-i}=\left[\frac{i}{n+2} \bar{c}_{3}-\frac{n-i+1}{n+2} D_{3}^{1} \bar{c}_{1}-\frac{1}{n+2} D_{3}^{2} \bar{c}_{2}\right]\left(c^{3}\right)^{i}\left(\bar{c}_{1}\right)^{n-i} .
$$


Upon integrating by parts with respect to the harmonic derivatives $D_{3}^{1}$ and $D_{3}^{2}$, this expression is replaced by

$$
\frac{i}{n+2}\left(\bar{c}_{1}\right)^{n-i}\left(c^{3}\right)^{i-1}
$$

Similarly, in the last line of (A.9) we apply the identity

$$
c^{1}\left(\bar{c}_{1}\right)^{n-i}\left(c^{3}\right)^{i}=\left[\frac{n-i}{n+2} c^{1}+\frac{1}{n+2} D_{2}^{1} c^{2}+\frac{i+1}{n+2} D_{3}^{1} c^{3}\right]\left(c_{1}\right)^{n-i}\left(c^{3}\right)^{i}
$$

and again integrate by parts with respect to the harmonic derivatives. As a result, the expression $c^{1}\left(\bar{c}_{1}\right)^{n-i}\left(c^{3}\right)^{i}$ in (A.9) produces the term

$$
\frac{n-i}{n+2}\left(c^{3}\right)^{i}\left(\bar{c}_{1}\right)^{n-i-1} .
$$

Taking all this into account, the variation (A.9) can be written as

$$
\begin{aligned}
\delta_{\mathrm{sc}} \Gamma_{n}= & \int d \zeta\left(\begin{array}{l}
33 \\
11
\end{array}\right) d u\left(\bar{\omega}^{12} \omega_{23}\right)^{2} \sum_{i=0}^{n} \alpha_{i, n-i}[(i+2) \bar{A}+(n-i+2) A]\left(\bar{\omega}^{12} c^{3}\right)^{i}\left(\omega_{23} \bar{c}_{1}\right)^{n-i} \\
& +\int d \zeta\left(\begin{array}{l}
33 \\
11
\end{array}\right) d u\left(\bar{\omega}^{12} \omega_{23}\right)^{2} \sum_{i=1}^{n} \alpha_{i, n-i} \frac{i(i+2)}{n+2} \bar{A}\left(\bar{\omega}^{12} c^{3}\right)^{i-1}\left(\omega_{23} \bar{c}_{1}\right)^{n-i} \\
& +\int d \zeta\left(\begin{array}{l}
33 \\
11
\end{array}\right) d u\left(\bar{\omega}^{12} \omega_{23}\right)^{2} \sum_{i=0}^{n-1} \alpha_{i, n-i} \frac{(n-i)(n-i+2)}{n+2} A\left(\bar{\omega}^{12} c^{3}\right)^{i}\left(\omega_{23} \bar{c}_{1}\right)^{n-i-1}
\end{aligned}
$$

We observe that the terms in the last two lines in (A.15) cancel similar terms in the first line of $\delta_{\mathrm{sc}} \Gamma_{n-1}$, provided the coefficients $\alpha_{i j}$ obey the following two equations

$$
\begin{aligned}
& \alpha_{i, n-i} \frac{(n-i+2)(n-i)}{n+2}+\alpha_{i+1, n-i-1} \frac{(i+3)(i+1)}{n+2}=-(n+3) \alpha_{i, n-i-1}, \\
& \alpha_{i, n-i} \frac{(n-i+2)(n-i)}{n+2}-\alpha_{i+1, n-i-1} \frac{(i+3)(i+1)}{n+2}=-(n-2 i-1) \alpha_{i, n-i-1} .
\end{aligned}
$$

As a consequence, any two adjacent coefficients are related as

$$
\frac{\alpha_{i, j}}{\alpha_{i, j-1}}=-\frac{(j+1)(i+j+2)}{(j+2) j} .
$$

The solution of this equation is just (3.19).

\section{B Harmonic integrals}

The standard definition of the integration over the $\mathrm{SU}(3)$ harmonic variables reads [2]

$$
\int d u 1=1, \quad \int d u(\text { non-singlet } \mathrm{SU}(3) \text { irreducible representation })=0 .
$$

From this definition one can derive the following simple relations

$$
\int d u u_{i}^{1} \bar{u}_{1}^{j}=\int d u u_{i}^{3} \bar{u}_{3}^{j}=\frac{1}{3} \delta_{i}^{j}, \quad \int d u u_{i}^{1} \bar{u}_{1}^{j} u_{k}^{1} \bar{u}_{1}^{l}=\frac{1}{6} \delta_{i}^{(j} \delta_{k}^{l)}, \quad \text { etc. }
$$


All these integrals appear as particular cases of the following general formula

$$
\begin{aligned}
\int d u u_{i_{1}}^{1} \bar{u}_{1}^{i_{1}^{\prime}} \ldots u_{i_{n}}^{1} \bar{u}_{1}^{i_{n}^{\prime}} u_{j_{1}}^{3} \bar{u}_{3}^{j_{1}^{\prime}} \ldots u_{j_{m}}^{3} \bar{u}_{3}^{j_{m}^{\prime}}= & \sum_{k=0}^{m} \frac{2 m !(-1)^{k}}{(m+1)(k+n+2)(k+n+1) k !(m-k) !} \\
& \times \delta_{i_{1}}^{\left(i_{1}^{\prime}\right.} \ldots \delta_{i_{n}}^{i_{n}^{\prime}} \delta_{\left(j_{1}\right.}^{\left\{j_{1}^{\prime}\right.} \ldots \delta_{j_{k}}^{\left.j_{k}^{\prime}\right)} \ldots \delta_{\left.j_{m}\right)}^{\left.j_{m}^{\prime}\right\}} .
\end{aligned}
$$

Here both $(\ldots)$ and $\{\ldots\}$ denote symmetrization of the indices. Contracting this expression with vev constants $c^{i}, \bar{c}_{i}$ and with scalar fields $\phi^{i}, \bar{\phi}_{i}$ we find

$$
\begin{aligned}
\int d u\left(\phi^{1} \bar{c}_{1}\right)^{n}\left(c^{3} \bar{\phi}_{3}\right)^{m}= & \sum_{k=0}^{m} \frac{2 m !(-1)^{k}}{(m+1)(k+n+2)(k+n+1) k !(m-k) !} \\
& \times \phi^{\left(i_{1}\right.} \ldots \phi^{i_{n}} c^{j_{1}} \ldots c^{\left.j_{k}\right)} \ldots c^{j_{m}} \bar{c}_{i_{1}} \ldots \bar{c}_{i_{n}} \bar{\phi}_{j_{1}} \ldots \bar{\phi}_{j_{k}} \ldots \bar{\phi}_{j_{m}} .
\end{aligned}
$$

After applying some combinatorics, this expression can be represented in the following useful form

$$
\begin{aligned}
\int d u\left(\phi^{1} \bar{c}_{1}\right)^{n}\left(c^{3} \bar{\phi}_{3}\right)^{m}= & \sum_{k=0}^{\min (m, n)} \frac{2 n ! m !(m+n-k+1) !(-1)^{k}}{k !(n-k) !(m-k) !(m+n+2) !(n+1)(m+1)} \\
& \times\left(\phi^{i} \bar{\phi}_{i}\right)^{k}\left(\phi^{i} \bar{c}_{i}\right)^{n-k}\left(c^{i} \bar{\phi}_{i}\right)^{m-k} .
\end{aligned}
$$

\section{Wess-Zumino term}

\section{C.1 Derivation from five-dimensions}

Consider six real scalar fields $X^{A}, A=1, \ldots, 6$, in the fundamental representation of $\mathrm{SO}(6)$. The WZ term for these scalars has the standard form $[22,23]$

$$
S_{W Z}=-\frac{1}{60 \pi^{2}} \int d^{5} x \varepsilon^{M N K L P} \varepsilon^{A B C D E F} \frac{1}{|X|^{6}} X^{A} \partial_{M} X^{B} \partial_{N} X^{C} \partial_{K} X^{D} \partial_{L} X^{E} \partial_{P} X^{F},
$$

where $|X|^{2}=X^{A} X^{A}$. It is useful to introduce the normalized scalars,

$$
Y^{A}=\frac{X^{A}}{|X|}, \quad Y^{A} Y^{A}=1
$$

in terms of which the action (C.1) can be rewritten as

$$
S_{W Z}=-\frac{1}{60 \pi^{2}} \int d^{5} x \varepsilon^{M N K L P} \varepsilon^{A B C D E F} Y^{A} \partial_{M} Y^{B} \partial_{N} Y^{C} \partial_{K} Y^{D} \partial_{L} Y^{E} \partial_{P} Y^{F} .
$$

The integration here is performed over a five-dimensional manifold $\mathcal{M}$ which has the fourdimensional Minkowski space as its boundary $\partial \mathcal{M}$.

Let us rewrite the action (C.3) in the manifestly SU(3) covariant form. For this purpose we pass to the complex scalars,

$$
\begin{aligned}
& f^{1}=Y^{1}+i Y^{2}, \quad f^{2}=Y^{3}+i Y^{4}, \quad f^{3}=Y^{5}+i Y^{6}, \\
& \bar{f}_{1}=Y^{1}-i Y^{2}, \quad \bar{f}_{2}=Y^{3}-i Y^{4}, \quad \bar{f}_{3}=Y^{5}-i Y^{6} \text {, }
\end{aligned}
$$


which are also normalized,

$$
f^{i} \bar{f}_{i}=1 .
$$

In terms of these scalars the action (C.3) acquires the desired manifestly SU(3) covariant form,

$$
\begin{aligned}
S_{W Z}= & \frac{i}{48 \pi^{2}} \varepsilon^{M N K L P} \varepsilon_{i j k} \varepsilon^{i^{\prime} j^{\prime} k^{\prime}} \int d^{5} x\left[-\left(f^{i} \partial_{M} f^{j} \partial_{N} f^{k}\right) \partial_{K}\left(\bar{f}_{l} \partial_{L} \bar{f}_{m} \partial_{P} \bar{f}_{n}\right)\right. \\
& \left.+\partial_{K}\left(f^{i} \partial_{M} f^{j} \partial_{N} f^{k}\right)\left(\bar{f}_{i^{\prime}} \partial_{L} \bar{f}_{j^{\prime}} \partial_{P} \bar{f}_{k^{\prime}}\right)\right] .
\end{aligned}
$$

It is also useful to rewrite (C.6) as

$$
S_{W Z}=\frac{i}{48 \pi^{2}} \int_{\mathcal{M}}\left(d \omega_{2} \wedge \bar{\omega}_{2}-\omega_{2} \wedge d \bar{\omega}_{2}\right)
$$

where $\omega_{2}$ and $\bar{\omega}_{2}$ are 2 -forms,

$$
\omega_{2}=\varepsilon_{i j k} f^{i} d f^{j} \wedge d f^{k}, \quad \bar{\omega}_{2}=\varepsilon^{i j k} \bar{f}_{i} d \bar{f}_{j} \wedge d \bar{f}_{k} .
$$

Note that the action (C.7) is real.

The equation (C.5) has the evident consequence

$$
d f^{i} \bar{f}_{i}+f^{i} d \bar{f}_{i}=0 .
$$

Using this relation, it is easy to prove the following important identity

$$
\omega_{2} \wedge d \bar{\omega}_{2}=-d \omega_{2} \wedge \bar{\omega}_{2}
$$

or

$$
d\left(\omega_{2} \wedge \bar{\omega}_{2}\right)=0 .
$$

With taking into account this identity, the WZ action (C.7) acquires the form

$$
S_{W Z}=\frac{i}{24 \pi^{2}} \int_{\mathcal{M}} d \omega_{2} \wedge \bar{\omega}_{2} .
$$

Now, let us introduce the projections

$$
y=f^{i} \bar{c}_{i}, \quad \bar{y}=\bar{f}_{i} c^{i},
$$

where $c^{i}$ are arbitrary constants with the SU(3) index. Owing to the identities

$$
d y \wedge \omega_{2}=\frac{y}{3} d \omega_{2}, \quad d \bar{y} \wedge \bar{\omega}_{2}=\frac{\bar{y}}{3} d \bar{\omega}_{2},
$$

the WZ action (C.7) can be rewritten as

$$
S_{W Z}=\frac{i}{8 \pi^{2}} \int_{\mathcal{M}} \frac{1}{y} d y \wedge \omega_{2} \wedge \bar{\omega}_{2}=\frac{i}{8 \pi^{2}} \int_{\mathcal{M}} d \ln y \wedge \omega_{2} \wedge \bar{\omega}_{2},
$$

or, in the self-conjugated form,

$$
S_{W Z}=\frac{i}{16 \pi^{2}} \int_{\mathcal{M}} d \ln \frac{y}{\bar{y}} \wedge \omega_{2} \wedge \bar{\omega}_{2} .
$$


Due to the identity (C.11), it is easy to integrate this form and to rewrite $S_{W Z}$ as an integral over the $d=4$ Minkowski boundary

$$
S_{W Z}=\frac{i}{16 \pi^{2}} \int_{\mathcal{M}} d\left[\ln \frac{y}{\bar{y}} \omega_{2} \wedge \bar{\omega}_{2}\right]=\frac{i}{16 \pi^{2}} \int_{\partial \mathcal{M}} \ln \frac{y}{\bar{y}} \omega_{2} \wedge \bar{\omega}_{2}+\chi_{4},
$$

where $\chi_{4}$ is an arbitrary closed 4 -form, $d \chi_{4}=0$. For simplicity, in what follows we assume that $\chi_{4}=0$, but, in general, there is no any prescription how to fix this 4 -form.

In terms of the scalars (C.4), the action (C.17) can be cast in the following explicit form,

$$
S_{W Z}=\frac{i}{16 \pi^{2}} \varepsilon^{m n p q} \varepsilon_{i j k} \varepsilon^{i^{\prime} j^{\prime} k^{\prime}} \int d^{4} x \ln \frac{f^{l} \bar{c}_{l}}{\bar{f}_{l^{\prime}} c^{l^{\prime}}}\left(f^{i} \partial_{m} f^{j} \partial_{n} f^{k}\right)\left(\bar{f}_{i^{\prime}} \partial_{p} \bar{f}_{j^{\prime}} \partial_{q} \bar{f}_{k^{\prime}}\right) .
$$

It is now easy to come back to the non-normalized scalars, $f^{i}=\varphi^{i} / \sqrt{\varphi^{l} \bar{\varphi}_{l}}, \bar{f}_{i}=\bar{\varphi}_{i} / \sqrt{\varphi^{l} \bar{\varphi}_{l}}$ and to obtain the final four-dimensional form of the WZ action:

$$
S_{W Z}=\frac{i}{16 \pi^{2}} \varepsilon^{m n p q} \varepsilon_{i j k} \varepsilon^{i^{\prime} j^{\prime} k^{\prime}} \int d^{4} x \ln \frac{\varphi^{l} \bar{c}_{l}}{\bar{\varphi}_{l^{\prime}} c^{l^{\prime}}} \frac{\left(\varphi^{i} \partial_{m} \varphi^{j} \partial_{n} \varphi^{k}\right)\left(\bar{\varphi}_{i^{\prime}} \partial_{p} \bar{\varphi}_{j^{\prime}} \partial_{q} \bar{\varphi}_{k^{\prime}}\right)}{\left(\varphi^{i} \bar{\varphi}_{i}\right)^{3}} .
$$

The constants $c^{i}$ in this action break the explicit SU(3) invariance. Nevertheless, (C.19) is $\mathrm{SU}(3)$ invariant (up to total derivatives), since it was derived from the $\mathrm{SU}(3)$ covariant five-dimensional action (C.6). The same argument implies that (C.19) respects a hidden $\mathrm{SO}(6) \sim \mathrm{SU}(4)$ invariance.

\section{C.2 Expansion around vacuum}

We point out that the constants $c^{i}$ in (C.19) are arbitrary. In this subsection we show that this action can appear in the component field expansion of the superfield action (3.16) if the constants $c^{i}$ coincide with the vevs of the scalars.

Let us assume that the constants $c^{i}$ in (C.19) are given by (3.8) and make the series expansion of this action around these vevs,

$$
\begin{aligned}
S_{W Z}= & \frac{i}{16 \pi^{2}} \varepsilon^{m n p q} \varepsilon_{i j k} \varepsilon^{i^{\prime} j^{\prime} k^{\prime}} \int d^{4} x\left(c^{i}+\phi^{i}\right) \partial_{m} \phi^{j} \partial_{n} \phi^{k}\left(\bar{c}_{i^{\prime}}+\bar{\phi}_{i^{\prime}}\right) \partial_{p} \bar{\phi}_{j^{\prime}} \partial_{q} \bar{\phi}_{k^{\prime}} \\
& \times \sum_{l=1}^{\infty} \frac{(-1)^{l+1}}{l}\left[\left(\phi^{i} \bar{c}_{i}\right)^{l}-\left(c^{i} \bar{\phi}_{i}\right)^{l}\right] \frac{1}{2} \sum_{m, n, k=0}^{\infty} \frac{(m+n+k+2) !}{m ! n ! k !}\left(c^{i} \bar{\phi}_{i}\right)^{m}\left(\bar{c}_{i} \phi^{i}\right)^{n}\left(\phi^{i} \bar{\phi}_{i}\right)^{k} .
\end{aligned}
$$

Here the fields $\phi^{i}$ are related with $\varphi^{i}$ as in (3.11) and we assume that $c^{i} \bar{c}_{i}=1$.

Let us single out in the series (C.20) the terms with minimal numbers of fields $\phi^{i}$ and $\bar{\phi}_{i}$. These terms correspond to the choice $m=n=0$ and $l=1$ in the second line in (C.20),

$$
S_{W Z}=\frac{i}{16 \pi^{2}} \varepsilon^{m n p q} \varepsilon_{i j k} \varepsilon^{i^{\prime} j^{\prime} k^{\prime}} \int d^{4} x\left(\phi^{l} \bar{c}_{l}-c^{l} \bar{\phi}_{l}\right) c^{i} \partial_{m} \phi^{j} \partial_{n} \phi^{k} \bar{c}_{i^{\prime}} \partial_{p} \bar{\phi}_{j^{\prime}} \partial_{q} \bar{\phi}_{k^{\prime}}+O\left(\phi^{6}\right) .
$$

Up to total derivatives, the following identity holds:

$$
\begin{aligned}
& \varepsilon^{m n p q} \varepsilon_{i j k} \varepsilon^{i^{\prime} j^{\prime} k^{\prime}}\left(\phi^{l} \bar{c}_{l}-c^{l} \bar{\phi}_{l}\right) c^{i} \partial_{m} \phi^{j} \partial_{n} \phi^{k} \bar{c}_{i^{\prime}} \partial_{p} \bar{\phi}_{j^{\prime}} \partial_{q} \bar{\phi}_{k^{\prime}} \\
= & \frac{1}{3} \varepsilon^{m n p q} \varepsilon_{i j k} \varepsilon^{i^{\prime} j^{\prime} k^{\prime}}\left(\phi^{i} \partial_{m} \phi^{j} \partial_{n} \phi^{k} \bar{c}_{i^{\prime}} \partial_{p} \bar{\phi}_{j^{\prime}} \partial_{q} \bar{\phi}_{k^{\prime}}-c^{i} \partial_{m} \phi^{j} \partial_{n} \phi^{k} \bar{\phi}_{i^{\prime}} \partial_{p} \bar{\phi}_{j^{\prime}} \partial_{q} \bar{\phi}_{k^{\prime}}\right)+\text { tot. deriv. }
\end{aligned}
$$


Using it, the action (C.21) can be written as

$$
\begin{gathered}
S_{W Z}=\frac{i}{48 \pi^{2}} \varepsilon^{m n p q} \varepsilon_{i j k} \varepsilon^{i^{\prime} j^{\prime} k^{\prime}} \int d^{4} x\left(\phi^{i} \partial_{m} \phi^{j} \partial_{n} \phi^{k} \bar{c}_{i^{\prime}} \partial_{p} \bar{\phi}_{j^{\prime}} \partial_{q} \bar{\phi}_{k^{\prime}}\right. \\
\left.-c^{i} \partial_{m} \phi^{j} \partial_{n} \phi^{k} \bar{\phi}_{i^{\prime}} \partial_{p} \bar{\phi}_{j^{\prime}} \partial_{q} \bar{\phi}_{k^{\prime}}\right)+O\left(\phi^{6}\right) .
\end{gathered}
$$

The assumption that $c^{i}$ in (C.19) coincide with the vevs was essential in deriving this expression. In Sect. 4.3 we showed that precisely these terms follow from the $\mathcal{N}=3$ superfield action (3.16).

\section{Effective equations of motion}

Effective actions in quantum field theory can be used to obtain effective equations of motion which describe the dynamics of fields with taking account of quantum corrections. For this purpose an effective action should be well defined off the classical mass shell, but this is not always possible. For instance, in [35, 36, 38] the constrained $\mathcal{N}=4$ superfields were used for constructing the $\mathcal{N}=4$ SYM low-energy effective action. Such on-shell effective actions should be treated rather in the S-matrix sense, but they cannot be used for obtaining the effective equations of motion. One of the merits of the $\mathcal{N}=3$ harmonic superspace approach is the possibility to relax the on-shell constraints for the superfield strengths and to express them in terms of unconstrained gauge superfield potentials. As a result, we are able to derive the superfield equations of motion which follow from the effective action (3.16).

Recall that the superfield strengths (2.15) are expressed in terms on the non-analytic gauge prepotentials $V_{1}^{2}$ and $V_{2}^{3}$ which, in turn, are related to the analytic ones by the zero-curvature equations (2.14). This is completely analogous to the $\mathcal{N}=2$ harmonic superspace approach [2] in which the chiral superfield strength $W$ has a simple differential expression in terms of the non-analytic gauge prepotential $V^{--}$which, in turn, is related to the analytic gauge potential $V^{++}$via the zero-curvature equation $D^{++} V^{--}=D^{--} V^{++}$. The solution of this equation is known to be non-local with respect to the harmonic variables $[39,40]$ and to involve some harmonic distributions. In our case the solutions of (2.14) are also non-local in the harmonic variables, but we avoid using the harmonic distributions by representing the solutions of (2.14) in the pseudo-differential form,

$$
V_{1}^{2}=D_{1}^{2} D_{1}^{2} \frac{1}{2+D_{1}^{2} D_{2}^{1}} V_{2}^{1}, \quad V_{2}^{3}=D_{2}^{3} D_{2}^{3} \frac{1}{2+D_{2}^{3} D_{3}^{2}} V_{3}^{2},
$$

where

$$
\frac{1}{2+D_{1}^{2} D_{2}^{1}}=\frac{1}{2} \sum_{n=0}^{\infty}\left(-\frac{1}{2} D_{1}^{2} D_{2}^{1}\right)^{n}, \quad \frac{1}{2+D_{2}^{3} D_{3}^{2}}=\frac{1}{2} \sum_{n=0}^{\infty}\left(-\frac{1}{2} D_{2}^{3} D_{3}^{2}\right)^{n} .
$$

The formal expressions (D.1) can be verified to obey (2.14) by making use of the commutation relations between the harmonic derivatives,

$$
\left[D_{2}^{1}, D_{1}^{2}\right]=S_{1}, \quad\left[D_{3}^{2}, D_{2}^{3}\right]=S_{2},
$$


where $S_{1}$ and $S_{2}$ are commuting U(1) generators in the su(3) algebra of harmonic derivatives. The gauge potentials have the following charges with respect to these $\mathrm{U}(1)$ generators,

$$
S_{1} V_{2}^{1}=2 V_{2}^{1}, \quad S_{2} V_{3}^{2}=2 V_{3}^{2} .
$$

Combining (D.1) with (2.15), we obtain formal expressions of the superfield strengths in terms on the analytic gauge potentials,

$$
\bar{W}^{12}=-\frac{1}{4}\left(D^{1}\right)^{2} D_{1}^{2} D_{1}^{2} \frac{1}{2+D_{1}^{2} D_{2}^{1}} V_{2}^{1}, \quad W_{23}=\frac{1}{4}\left(\bar{D}_{3}\right)^{2} D_{2}^{3} D_{2}^{3} \frac{1}{2+D_{2}^{3} D_{3}^{2}} V_{3}^{2} .
$$

Using the equation (3.25) for the function $H$, one can easily compute the variation of the action (3.16),

$$
\delta \Gamma=\frac{\alpha}{8} \int d \zeta\left(\begin{array}{l}
33 \\
11
\end{array}\right) d u \frac{\delta \bar{\omega}^{12} \bar{\omega}^{12} \omega_{23} \omega_{23}}{\left(1+\bar{\omega}^{12} c^{3}\right)\left(1+\bar{\omega}^{12} c^{3}+\omega_{23} \bar{c}_{1}\right)^{2}}+\text { c.c. . }
$$

Here we assume $c^{i} \bar{c}_{i}=1$ for simplicity. Owing to (D.5), the variation of the superfield strength $\bar{\omega}^{12}$ can be expressed through the variation of the analytic gauge potential $V_{2}^{1}$,

$$
\delta \bar{\omega}^{12}=-\frac{1}{4}\left(D^{1}\right)^{2} D_{1}^{2} D_{1}^{2} \frac{1}{2+D_{1}^{2} D_{2}^{1}} \delta V_{2}^{1} .
$$

Then the equation of motion produced by the variation of the action (3.16) with respect to $V_{2}^{1}$ reads

$$
\frac{\delta \Gamma}{\delta V_{2}^{1}}=-\frac{\alpha}{32} \frac{1}{2+D_{2}^{1} D_{1}^{2}} D_{1}^{2} D_{1}^{2}\left(D^{1}\right)^{2} \frac{\bar{\omega}^{12} \omega_{23} \omega_{23}}{\left(1+\bar{\omega}^{12} c^{3}\right)\left(1+\bar{\omega}^{12} c^{3}+\omega_{23} \bar{c}_{1}\right)^{2}} .
$$

Applying the tilde-conjugation to (D.8), one can obtain the second equation of motion $\delta \Gamma / \delta V_{3}^{2}$.

Note that the equation (D.8) and its conjugate should not be considered separately, but they should be added to the classical equations of motion associated with the original Chern-Simons type action of the $\mathcal{N}=3$ gauge theory [3, 4]. Together they form the effective equations of motion up to the four-derivative order.

\section{References}

[1] I. L. Buchbinder and S. M. Kuzenko, Ideas and methods of supersymmetry and supergravity: Or a walk through superspace, Bristol, UK: IOP (1998) 656 p.

[2] A. S. Galperin, E. A. Ivanov, V. I. Ogievetsky and E. S. Sokatchev, Harmonic superspace, Cambridge, UK: Univ. Pr. (2001) 306 p.

[3] A. Galperin, E. Ivanov, S. Kalitzin, V. Ogievetsky and E. Sokatchev, N=3 Supersymmetric gauge theory, Phys. Lett. B 151 (1985) 215.

[4] A. Galperin, E. Ivanov, S. Kalitzin, V. Ogievetsky and E. Sokatchev, Unconstrained off-shell N=3 supersymmetric Yang-Mills theory, Class. Quant. Grav. 2 (1985) 155.

[5] F. Delduc and J. McCabe, The quantization of N=3 super Yang-Mills off-shell in harmonic superspace, Class. Quant. Grav. 6 (1989) 233. 
[6] E. A. Ivanov and B. M. Zupnik, N=3 supersymmetric Born-Infeld theory, Nucl. Phys. B 618 (2001) 3, arXiv:hep-th/0110074.

[7] I. L. Buchbinder, E. A. Ivanov, I. B. Samsonov and B. M. Zupnik, Scale invariant low-energy effective action in N=3 SYM theory, Nucl. Phys. B 689 (2004) 91, arXiv:hep-th/0403053.

[8] M. Dine and N. Seiberg, Comments on higher derivative operators in some SUSY field theories, Phys. Lett. B 409 (1997) 239, arXiv:hep-th/9705057.

[9] N. Seiberg, Notes on theories with 16 supercharges, Nucl. Phys. Proc. Suppl. 67 (1998) 158, arXiv:hep-th/9705117.

[10] B. de Wit, M. T. Grisaru and M. Roček, Nonholomorphic corrections to the one-loop N=2 super Yang-Mills action, Phys. Lett. B 374 (1996) 297, arXiv:hep-th/9601115.

[11] V. Periwal and R. von Unge, Accelerating D-branes, Phys. Lett. B 430 (1998) 71, arXiv:hep-th/9801121.

[12] F. Gonzalez-Rey and M. Roček, Nonholomorphic N=2 terms in N=4 SYM: 1-loop calculation in N=2 superspace, Phys. Lett. B 434 (1998) 303, arXiv:hep-th/9804010.

[13] I. L. Buchbinder and S. M. Kuzenko, Comments on the background field method in harmonic superspace: Non-holomorphic corrections in N=4 SYM, Mod. Phys. Lett. A 13 (1998) 1623, arXiv: hep-th/9804168.

[14] F. Gonzalez-Rey, B. Kulik, I. Y. Park and M. Roček, Self-dual effective action of N=4 super-Yang-Mills, Nucl. Phys. B 544 (1999) 218, arXiv:hep-th/9810152.

[15] E. I. Buchbinder, I. L. Buchbinder and S. M. Kuzenko, Non-holomorphic effective potential in N=4 SU(n) SYM, Phys. Lett. B 446 (1999) 216, arXiv:hep-th/9810239.

[16] E. I. Buchbinder, B. A. Ovrut, I. L. Buchbinder, E. A. Ivanov, S. M. Kuzenko, Low-energy effective action in N=2 supersymmetric field theories, Phys. Part. Nucl. 32 (2001) 641-674.

[17] I. L. Buchbinder and E. A. Ivanov, Complete $N=4$ structure of low-energy effective action in $N=4$ superYang-Mills theories, Phys. Lett. B 524 (2002) 208, arXiv:hep-th/0111062.

[18] I. L. Buchbinder, E. A. Ivanov and A. Y. Petrov, Complete low-energy effective action in N=4 SYM: A Direct N=2 supergraph calculation, Nucl. Phys. B 653 (2003) 64, arXiv:hep-th/0210241.

[19] A. T. Banin, I. L. Buchbinder and N. G. Pletnev, One loop effective action for N=4 SYM theory in the hypermultiplet sector: Leading low-energy approximation and beyond, Phys. Rev. D 68 (2003) 065024, arXiv:hep-th/0304046.

[20] I. L. Buchbinder and N. G. Pletnev, Hypermultiplet dependence of one-loop effective action in the N=2 superconformal theories, JHEP 0704 (2007) 096, arXiv:hep-th/0611145.

[21] A. Galperin, E. Ivanov and V. Ogievetsky, Superspace actions and duality transformations for N=2 tensor multiplets, Sov. J. Nucl. Phys. 45 (1987) 157 [Yad. Fiz. 45 (1987) 245] [Phys. Scripta T15 (1987) 176].

[22] A. A. Tseytlin and K. Zarembo, Magnetic interactions of D-branes and Wess-Zumino terms in super Yang-Mills effective actions, Phys. Lett. B 474 (2000) 95, arXiv:hep-th/9911246.

[23] K. A. Intriligator, Anomaly matching and a Hopf-Wess-Zumino term in 6d, N=(2,0) field theories, Nucl. Phys. B 581 (2000) 257, arXiv:hep-th/0001205. 
[24] L. Andrianopoli, S. Ferrara, E. Sokatchev and B. Zupnik, Shortening of primary operators in $N$ extended SCFT(4) and harmonic superspace analyticity, Adv. Theor. Math. Phys. 4 (2000) 1149, arXiv: hep-th/9912007.

[25] P. S. Howe, K. S. Stelle and P. K. Townsend, Supercurrents, Nucl. Phys. B 192 (1981) 332.

[26] I. L. Buchbinder and I. B. Samsonov, N=3 Superparticle model, Nucl. Phys. B 802 (2008) 180, arXiv:0801.4907 [hep-th].

[27] A. S. Galperin, E. A. Ivanov, V. I. Ogievetsky, Superspaces for $N=3$ supersymmetry, Sov. J. Nucl. Phys. 46 (1987) 543-556.

[28] R. R. Metsaev and A. A. Tseytlin, Supersymmetric D3-brane action in $A d S_{\times} S^{5}$, Phys. Lett. B 436 (1998) 281, arXiv:hep-th/9806095.

[29] I. Chepelev and A. A. Tseytlin, Long distance interactions of branes: Correspondence between supergravity and superYang-Mills descriptions, Nucl. Phys. B 515 (1998) 73, arXiv:hep-th/9709087.

[30] J. M. Maldacena, The Large N limit of superconformal field theories and supergravity, Adv. Theor. Math. Phys. 2 (1998) 231, arXiv: hep-th/9711200.

[31] E. Keski-Vakkuri and P. Kraus, Born-Infeld actions from matrix theory, Nucl. Phys. B 518 (1998) 212, arXiv:hep-th/9709122.

[32] S. P. de Alwis, Matrix models and string world sheet duality, Phys. Lett. B 423 (1998) 59, arXiv:hep-th/9710219.

[33] V. Balasubramanian, R. Gopakumar and F. Larsen, Gauge theory, geometry and the large N limit, Nucl. Phys. B 526 (1998) 415, arXiv:hep-th/9712077.

[34] A. A. Tseytlin, Born-Infeld action, supersymmetry and string theory, In *Shifman, M.A. (ed.): The many faces of the superworld* 417-452, arXiv:hep-th/9908105.

[35] D. V. Belyaev and I. B. Samsonov, Wess-Zumino term in the N=4 SYM effective action revisited, JHEP 1104 (2011) 112, arXiv:1103.5070 [hep-th].

[36] D. V. Belyaev and I. B. Samsonov, Bi-harmonic superspace for $N=4 d=4$ super Yang-Mills, JHEP 1109 (2011) 056, arXiv:1106.0611 [hep-th].

[37] B. de Wit, R. Philippe and A. Van Proeyen, The improved tensor multiplet in N=2 supergravity, Nucl. Phys. B 219 (1983) 143.

[38] I. L. Buchbinder, O. Lechtenfeld and I. B. Samsonov, $N=4$ superparticle and super Yang-Mills theory in USp(4) harmonic superspace, Nucl. Phys. B 802 (2008) 208, arXiv:0804.3063 [hep-th].

[39] B. M. Zupnik, The action of the supersymmetric N=2 gauge theory in harmonic superspace, Phys. Lett. B 183 (1987) 175.

[40] B. M. Zupnik, Six-dimensional supergauge theories in the harmonic superspace, Sov. J. Nucl. Phys. 44 (1986) 512 [Yad. Fiz. 44 (1986) 794]. 